Research Paper

\title{
Interplay of Oxidative Stress and Autophagy in PAMAM Dendrimers-Induced Neuronal Cell Death
}

\author{
Yubin Li 1\#, Haiyan Zhu 1\#, Shaofei Wang 1\#, Xiaolu Qian 2, Jiajun Fan 1, Ziyu Wang 1, Ping Song 1, Xuesai \\ Zhang ${ }^{3}$, Weiyue Lu ${ }^{4}$, Dianwen Ju ${ }^{1}{ }^{凶}$ \\ 1. Department of Biosynthesis \& The Key Laboratory of Smart Drug Delivery, Ministry of Education, School of Pharmacy, Fudan University, \\ Shanghai 201203, P.R. China \\ 2. Shanghai Animal Disease Control Center, Shanghai 201103, P.R. China \\ 3. Department of Pharmacology, School of Pharmacy, Fudan University, Shanghai 201203, P.R. China \\ 4. Department of Pharmaceutics \& The Key Laboratory of Smart Drug Delivery, Ministry of Education, School of Pharmacy, Fudan University, \\ Shanghai 201203, P.R. China \\ \# Yubin Li, Haiyan Zhu, and Shaofei Wang contributed equally to this work.
}

$\triangle$ Corresponding author: Dianwen Ju, Department of Biosynthesis \& The Key Laboratory of Smart Drug Delivery, Ministry of Education, School of Pharmacy, Fudan University, Shanghai 201203, P.R. China. Tel: +862151980037. Fax: +862151980036. Email: dianwenju@fudan.edu.cn

() 2015 Ivyspring International Publisher. Reproduction is permitted for personal, noncommercial use, provided that the article is in whole, unmodified, and properly cited. See http://ivyspring.com/terms for terms and conditions.

Received: 2015.07.07; Accepted: 2015.08.30; Published: 2015.10.08

\begin{abstract}
Poly-amidoamine (PAMAM) dendrimers are proposed to be one of the most promising drug-delivery nanomaterials. However, the toxicity of PAMAM dendrimers on the central nervous system seriously hinders their medical applications. The relationship between oxidative stress and autophagy induced by PAMAM dendrimers, and its underlying mechanism remain confusing. In this study, we reported that PAMAM dendrimers induced both reactive oxygen species and autophagy flux in neuronal cells. Interestingly, autophagy might be triggered by the formation of reactive oxygen species induced by PAMAM dendrimers. Suppression of reactive oxygen species could not only impair PAMAM dendrimers-induced autophagic effects, but also reduce PAMAM dendrimers-induced neuronal cell death. Moreover, inhibition of autophagy could protect against PAMAM dendrimers-induced neuronal cell death. These findings systematically elucidated the interplay between oxidative stress and autophagy in the neurotoxicity of PAMAM dendrimers, which might encourage the application of antioxidants and autophagy inhibitors to ameliorate the neurotoxicity of PAMAM dendrimers in clinic.
\end{abstract}

Key words: Oxidative stress, Autophagy, Nanomaterials, Neuronal cell

\section{Introduction}

Due to their unique chemical, mechanical and biological properties, functionalized nanomaterials designed for the commercial and medical applications have been widely researched, changing the prospect of pharmaceutical and biotechnology industries[1, 2]. Among them, poly-amidoamine (PAMAM) dendrimers are highly promising nanomaterials for therapeutic and diagnostic purposes[3-5]. Although PAMAM dendrimers have been developed as multifunctional therapeutic agents for anti-pathogen or anti-tumor treatments, the toxicological effects of
PAMAM dendrimers seriously limited their applications [6]. It has been confirmed that PAMAM dendrimers could promote acute lung injury, disrupt key platelet functions, initiate blood clot formation and induce toxic response in the central nervous system[7-10]. But to date, the scientific basis for the cytotoxicity of nanomaterials is not well elucidated, and understanding of the role and mechanism of toxicity induced by PAMAM dendrimers is still finite.

An important mechanism of nanotoxicity is the abnormal generation of reactive oxygen species 
(ROS)[11, 12]. A series of literatures have reported that oxidative stress triggered by overproduction of ROS could induce cellular damage and influence apoptotic or pro-inflammatory signaling pathway: Exposure to silver nanoparticles leads to the induction of ROS and apoptosis in mouse embryonic fibroblasts; Titanium nanoparticles induced renal fibrosis via oxidative stress upregulation; Titanium dioxide nanoparticles evoked strong oxidative stress and mitochondrial damage in glial cells; Zinc oxide nanoparticles could induce ROS generation by depleting antioxidant enzymes in macrophages[13-15]. Moreover, scientists have investigated that PAMAM dendrimers-induced cytotoxicity was triggered by ROS in mouse macrophage cells, while PEGylation could decrease PAMAM dendrimers-induced cytotoxicity via attenuation of oxidative stress[16, 17].

Macroautophagy (hereafter referred to as autophagy) is a self-digesting process that is implicated in multiple biological processes including cell death and differentiation, aging and neurodegenerative diseases, tumor occurrence and development[18]. Activation of autophagy requires formation of autophagosomes where eukaryotes degrade dysfunctional proteins and damaged organelles by lysosomal enzymatic content[19]. Characterized by the accumulation of autophagosomes and autophagolysosomes in the cytoplasm, autophagy has been classified as a new morphological form of programmed cell death[20]. Previous studies have reported that a variety of nanoparticles such as silica, ceria, quantum dots, and silver nanoparticles could trigger autophagy in various cell lines[21-23]. Researchers found that zinc oxide nanoparticles induced ROS generation in macrophages and simultaneously induced autophagy and apoptosis, indicating that autophagy might be a cellular defense mechanism against ROS[15]. We have also confirmed that PAMAM dendrimers could induce autophagy in human glioma cells[24], but the underlying relationship and mechanism between ROS and autophagy in PAMAM dendrimers-induced neuronal cell death are still unknown. A variety of evidence suggested that ROS were early inducers of autophagy upon nutrient deprivation[25]; meanwhile, ROS were also involved in the process of autophagy and regulated by autophagy under other pathological conditions such as brain injury and tumor. As mitochondria were main source of ROS in autophagy signaling[26], mitophagy became the major breakthrough to link ROS and autophagy, with series of molecular mechanisms underlying mitophagy characterized[27]. Besides, ROS could modify DNA and induce DNA damage; when DNA became unrepaired and apoptosis was defective, DNA damage-triggered autophagy contributed to cell death[28]. Thus, eluci- dating the interaction between ROS and autophagy would facilitate the management of PAMAM dendrimers-induced nanotoxicity.

The primary aim of the study was to explore the relationship between oxidative stress and autophagy in neurotoxicity induced by PAMAM dendrimers and to develop the new mechanism of PAMAM dendrimers-induced neurotoxicity. In the current research, we studied the generation of ROS and autophagy flux in pheochromocytoma cell line PC-12 and human neuroblastoma cell line SH-SY5Y treated with PAMAM dendrimers. Antioxidant $\mathrm{N}$-acetyl cysteine (NAC), lipoic acid (LA), and tocopherol were used to gain a preliminary insight into the cellular response (autophagic and cytotoxicological effects) to PAMAM dendrimers exposure by regulating oxidative stress. To further investigate the interplay between oxidative stress and autophagy in PAMAM dendrimers-induced neuronal cell death, the effects of PAMAM dendrimers combined with autophagy inhibitors (LY294002 and chloroquine) or autophagy activators (trehalose and rapamycin) were also examined in PC-12 cells. This study systematically elucidated the relationship between oxidative stress and autophagy in the neurotoxicity triggered by PAMAM dendrimers and suggested the application of antioxidants and autophagy inhibitors in ameliorating PAMAM dendrimers-induced neurotoxicity.

\section{Materials and Methods}

\section{Materials}

The PI3K inhibitor LY294002 was obtained from Beyotime Institute of Biotechnology (Haimen, China). The lysosomal inhibitor chloroquine (CQ) and autophagy inducer trehalose were obtained from Sigma (St Louis, MO). Antioxidants $\mathrm{N}$-acetyl cysteine (NAC), lipoic acid (LA), and tocopherol were purchased from Beyotime Institute of Biotechnology (Haimen, China). Bafilomycin A1 and Rapamycin were obtained from Sangon Biotech (Shanghai, China). Cyto-ID Green dye kit was purchased from ENZO Life Science (Farmingdale, NY, USA). MitoSox Red mitochondrial superoxide indicator was obtained from Life Technologies (Eugene, Oregon, USA). MEK 1/2 inhibitor U0126 was supplied by Cell Signaling Technology (Danvers, MA, USA). The antibodies used were as follows: anti-LC3B, anti-SQSTEM1/p62, anti-phospho-AKT (Ser473), anti-phospho-MTOR (Ser2448), anti-p70 S6 Kinase Phospho (pS371), anti-phospho-p44/42 MAPK (Erk 1/2) (Thr202/Tyr204), anti-tubulin were purchased from Cell Signaling Technology (Danvers, MA, USA); the second antibodies horseradish peroxidase (HRP)-conjugated goat anti-mouse and anti-rabbit immunoglobulin G (IgG) 
were obtained from Jackson ImmunoResearch Laboratories (West Grove, PA, USA). All cell culture reagents were purchased from Gibco (Carlsbad, CA, USA).

\section{Preparation of PAMAM dendrimers}

PAMAM dendrimers G5 dissolved in methanol solution was obtained from Sigma-Aldrich (St. Lous, $\mathrm{MO})$. For experiment use, PAMAM dendrimers were air dried, and then dissolved in $0.01 \mathrm{M}$ phosphate-buffered saline (PBS) (pH7.4)[29].

\section{Characteristics of PAMAM dendrimers G5}

The size distributions and zeta potentials of PAMAM dendrimers G5 were measured by photon correlation spectroscopy (3000HS; Malvern Instruments, Malvern, UK). The measurements were performed on $20 \mu \mathrm{M}$ of PAMAM dendrimers solutions at $23^{\circ} \mathrm{C}$ in methanol, Milli $\mathrm{Q}$ distilled water, PBS, RPMI-1640, or MEM/F-12 medium. Each sample was detected at least three times. UV/Visible absorption spectroscopic analyses of PAMAM dendrimers G5 solutions in PBS, RPMI-1640, or MEM/F-12 medium were performed by a Perkin Elmer Lambda 900 UV/visible/NIR absorption spectrometer. The spectroscopic measurements were performed on $15 \mu \mathrm{M}$ of PAMAM dendrimers solutions.

\section{Cell culture}

The rat pheochromocytoma cell line PC-12 and human neuroblastoma cell line SH-SY5Y were supplied by Cell Bank of Shanghai Institutes for Biological Science, Chinese Academy of Science (Shanghai, China). PC-12 cells were maintained in RPMI-1640 medium while SH-SY5Y cells were maintained in MEM/F-12 medium. All medium were supplemented with $10 \%$ heat-inactivated fetal bovine serum (Invitrogen, San Diego, CA, USA), 2mM L-glutamine, 100 $\mathrm{U} / \mathrm{mL}$ of penicillin, and $100 \mu \mathrm{g} / \mathrm{mL}$ of streptomycin (Beyotime Institute of Biotechnology, Haimen, China). All cells were cultured continuously at $37^{\circ} \mathrm{C}$ in an incubator with $95 \%$ air and $5 \% \mathrm{CO}_{2}$.

\section{Cell viability assay}

Cell viability assay was measured by Cell Counting Kit-8 (CCK-8) (Dojin Laboratories, Kumamoto, Japan). Briefly, cells were plated and incubated in 96-well plates at a density of $1 \times 10^{4}$ cells/well for $24 \mathrm{~h}$. When reaching $80 \% \sim 90 \%$ confluence, cells were treated with different concentrations of PAMAM dendrimers G5 for another $24 \mathrm{~h}$. The plates were incubated with CCK-8 in an atmosphere of air containing $5 \% \mathrm{CO}_{2}$ at $37^{\circ} \mathrm{C}$ for $30 \mathrm{~min}$, and then the absorbance was measured at $450 \mathrm{~nm}$.

\section{Western blot analysis}

Cells were washed with cold PBS, and kept in RIPA lysis buffer (Beyotime Institute of Biotechnology, Haimen, China) for $30 \mathrm{~min}$ on ice, and then the lysates were centrifuged to collect the supernatants. The concentration of protein was examined by the bicinchoninic acid (BCA) method. Equivalent amounts of protein were separated by sodium dodecyl sulfate-polyacrylaminde gelelectrophoresis (SDS-PAGE), and subsequently transferred to poly vinylidene fluoride membranes. The membranes were blocked in Tris-buffered saline and Tween 20 (TBST) containing $3 \%$ bovine serum albumin (BSA) for $1 \mathrm{~h}$ at room temperature, and then subjected to primary antibodies at $4{ }^{\circ} \mathrm{C}$ overnight. After incubation with the secondary antibody conjugated with horseradish peroxidase for $2 \mathrm{~h}$ at room temperature, membranes were washed in TBST and the signals were visualized using an enhanced chemiluminescent detection kit (Pierce, Rockford, IL).

\section{Confocal fluorescence assay}

$1 \times 10^{5}$ cells were cultured in dishes with glass bottom for $12 \mathrm{~h}$, and then treated with a series concentrations of PAMAM dendrimers G5 for different times. Cells were disposed with Cyto-ID Autophagy Detection Kit (ENZO Life Science, Farmingdale, NY, USA) and MitoSox Red mitochondrial superoxide indicator (Eugene, Oregon, USA) following the manufacturer's instruction [30]. The images were observed and overlaid by an inverted confocal microscope (Carl Zeiss LSM710, Carl Zeiss, Germany). Lysotracker Red (Invitrogen, San Diego, CA, USA) was used to stain lysosomes and autophagolysosomes. PC-12 cells were incubated with PAMAM dendrimers G5 for a series of times, then Cyto-ID Green Dye, LysoTracker Red, and Hoechst 33342 were used to stain cells at $37^{\circ} \mathrm{C}$ for $20 \mathrm{~min}$. After that, cells were washed in RPMI-1640 medium and observed by confocal microscopy immediately. All the relative fluorescence intensity was measure by inverted confocal microscope software (Carl Zeiss LSM710, Carl Zeiss, Germany).

\section{Reactive oxygen species assay}

Intracellular ROS generation was detected by reactive oxygen species assay kit (Beyotime Biotechnology, Haimen, China). Before experiment, $1 \times 10^{4}$ cells PC-12 cells were seeded in 96-well black plate per well for $24 \mathrm{~h}$. After series of indicated experimental treatments, cells were loaded with $10 \mu \mathrm{M}$ of fluorescent probe $2^{\prime}, 7^{\prime}$-dichlorofluorescein diacetate (DCFH-DA) in serum-free RPMI-1640 medium for 20 min at $37^{\circ} \mathrm{C}$. And then cells were washed thrice with 
serum-free medium. DCF fluorescence intensity was detected by Tecan Infinite 200 PRO microplate reader to quantify levels of ROS.

\section{Statistical analysis}

All the statistical analyses were measured by Graphpad Prism 5 (GraphPad Software Inc,. San Diego, CA). Data were presented as mean \pm standard deviations (SD) and analyzed by the two-tailed Student $t$ test. Levels of $p<0.05$ were considered as statistical significance.

\section{Results}

\section{Reactive oxygen species and autophagy were triggered by PAMAM dendrimers in neuronal cells.}

The characteristics and stability of PAMAM dendrimers G5 used in the experiment were detected in different media. As shown in Figure S1A, the average particle size of PAMAM dendrimers G5 in methanol, MQ water, PBS, RPMI-1640 medium, and MEM/F-12 medium were $5.45 \mathrm{~nm}, 5.53 \mathrm{~nm}, 6.02 \mathrm{~nm}$, $5.89 \mathrm{~nm}$, and $5.97 \mathrm{~nm}$, respectively; and was superior to those quoted by the manufacturer. The zeta potential of PAMAM dendrimers G5 in MQ water and PBS was $23.57 \mathrm{mV}$ and $21.30 \mathrm{mV}$, respectively; while the zeta potentials in RPMI-1640 medium and MEM/F-12 medium was $20.16 \mathrm{mV}$ and $20.79 \mathrm{mV}$, respectively (Figure S1B); due to the cationic surface amino group of PAMAM dendrimers G5. However, the zeta potentials in PC-12 cell culture medium and SHSY-5Y cell culture medium became $-6.27 \mathrm{mV}$ and $-3.78 \mathrm{mV}$. The lower zeta potential measured in PC-12 cell culture medium and SHSY-5Y cell culture medium suggested that the possible interplay between PAMAM dendrimers G5 and components in the media might induce PAMAM dendrimers aggregation as several literatures have ever reported[31]. To explore the dendrimers stability in RPMI-1640 medium and MEM/F-12 medium, the average particle sizes and zeta potentials of PAMAM dendrimers G5 in relevant cell culture media were detected at different time, and the data were shown in Figure S1C -S1E. Additionally, the UV/Visible absorption spectrum of the PBS media with PAMAM dendrimers G5 showed characteristic peaks at $\sim 210 \mathrm{~nm}$ and $\sim 290 \mathrm{~nm}$, while PAMAM dendrimers G5 in RPMI-1640 medium and MEM/F-12 medium also showed similar characteristic peaks at $\sim 210 \mathrm{~nm}$ and $\sim 290 \mathrm{~nm}$ (Figure S1F).

Scientists have noticed that intracellular reactive oxygen species are widely responsible for the toxicity of several nanomaterials[12], and the critical role of reactive oxygen species has been highlighted in the PAMAM dendrimers-induced toxic responses[31, 32].
As mitochondrion is the major organelle generating ROS, and oxidative damage is tightly associated with mitochondrial dysfunction[33, 34]; MitoSox Red dye (oxidative fluorescence) and Cyto-ID Green dye (autophagic fluorescence) were used to determine whether formation of ROS would contribute to PAMAM dendrimers-induced autophagy.

As shown in Figure 1, PAMAM dendrimers induced occurrence of oxidative fluorescence (red in web version) and accumulation of autophagic fluorescence (green in web version). The quantitative analysis of relative oxidative fluorescence and autophagic fluorescence intensity showed that PAMAM dendrimers induced continuous accumulation of oxidative fluorescence in the first $12 \mathrm{~h}$, with enhanced accumulation of autophagic fluorescence observed in the first 24h (Figure S2A and S2B). Oxidative fluorescence reached the highest peak prior to autophagic fluorescence and quenched earlier than autophagic fluorescence. Similar results were also shown in PAMAM dendrimers-treated SHSY-5Y cells (Figure S3).

These results suggested that PAMAM dendrimers induced both reactive oxygen species and autophagy in neuronal cells, while autophagy might be partly triggered by PAMAM dendrimers-induced reactive oxygen species in neuronal cells.

\section{Autophagic flux was induced by PAMAM den- drimers in neuronal cells.}

Autophagy is an evolutionarily conserved process through which cells recruit damaged proteins and dysfunctional organelles to autophagosomes and then fuse with lysosomes for their degradation under stress conditions; thus it is crucial to determine whether stimulus could promote autophagy or just block the fusion of autophagosomes and lysosomes[35, 36]. Cyto-ID Green dye and LysoTracker Red dye were used to explore whether autophagic flux was induced in PAMAM dendrimers-treated neuronal cells. As shown in Figure 2A, PAMAM dendrimers induced formation of autophagosomes (green fluorescence in the web version), fusion of autophagosomes with lysosomes (yellow fluorescence in the web version) and clearance of autophagosomes (red fluorescence in the web version) in a time-dependent manner. Similar results were also observed in PAMAM dendrimers-treated SHSY-5Y cells (Figure S4). Besides, a series of zoomed-in images with a z-stack also showed the co-localization of autophagosome-related fluorescence and lysosomes-related fluorescence in Figure S5. Furthermore, PAMAM dendrimers-induced autophagic flux was also monitored by Bafilomycin A1 treatment. Bafilomycin A1, an inhibitor of vacuo- 
lar-type ATPase, inhibits the fusion of autophagosomes with lysosomes in the late stage of autophagy through suppressing vesicle acidification. Although Bafilomycin A1 inhibits autophagosomes from fusing with lysosomes, it has no effects on the occurrence of autophagosomes[37]. As shown in Figure S6, after incubation with $10 \mu \mathrm{g} / \mathrm{mL}$ of PAMAM dendrimers for various times or with different concentrations of PAMAM dendrimers for $12 \mathrm{~h}$, additional treatment of cells with Bafilomycin A1 caused a further increased level of LC3-II and p62 (Figure 2B and 2C, Figure S7); while autophagy positive control rapamycin induced increased level of LC3-II and decreased level of p62 (Figure 2D and 2E). As p62 is an ubiquitin-binding protein that directly binds to LC3, it could be used as a marker to study autophagic flux. When autophagy is inhibited, p62 accumulates; when autophagy is induced, p62 decreases[29]. Thus, our results indicated that autophagic flux was induced in PAMAM dendrimers-treated PC-12 cells.

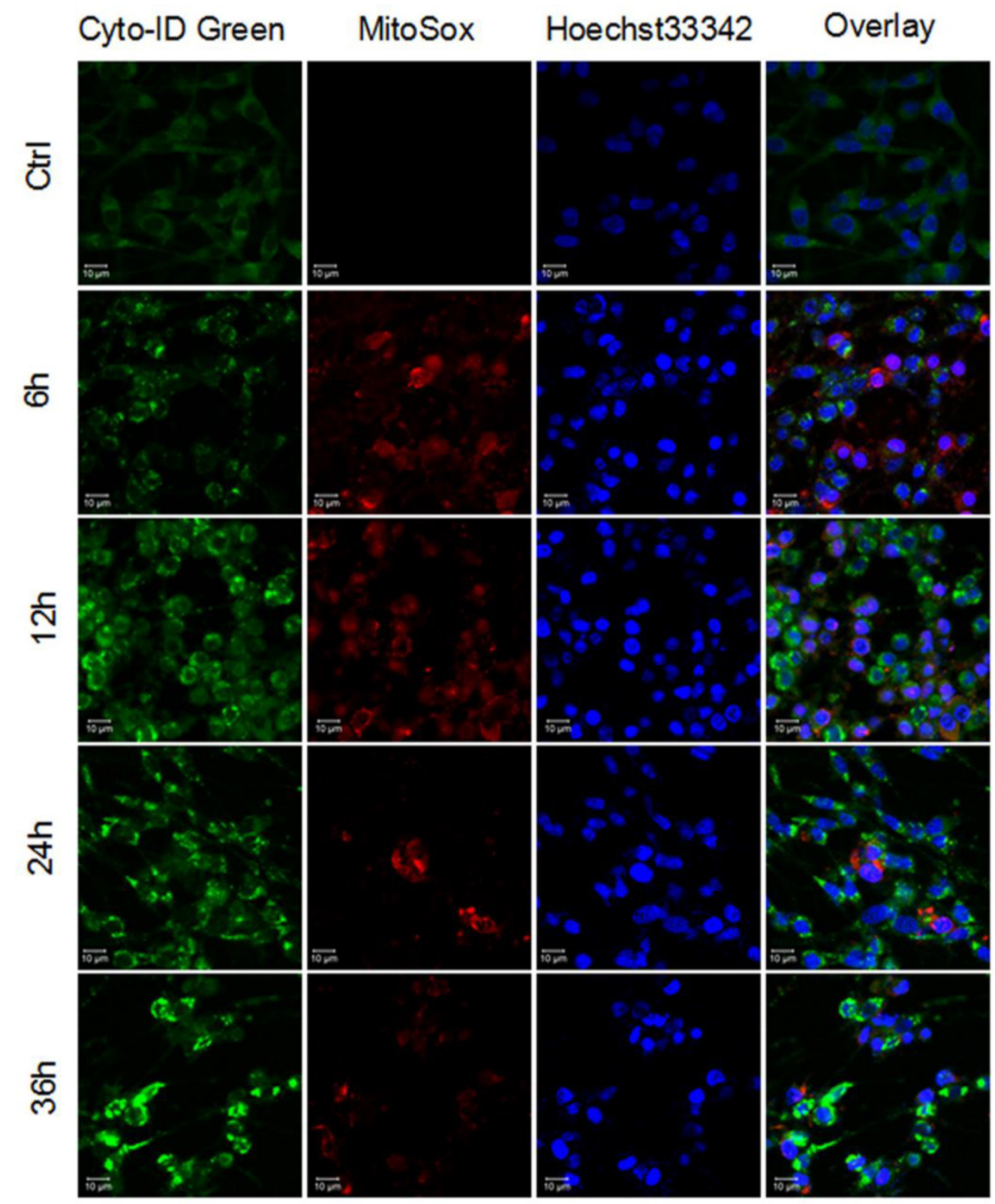

Figure 1. Reactive oxygen species and autophagy were triggered by PAMAM dendrimers in PC-12 cells. Cells were plated in cell culture dishes with glass bottom. After treated with $10 \mu \mathrm{g} / \mathrm{mL}$ of PAMAM dendrimers G5 for $6 \mathrm{~h}, 12 \mathrm{~h}, 24 \mathrm{~h}$, and $36 \mathrm{~h}$, cell samples concurrently stained with Cyto-ID Green dye and MitoSox Red dye were analyzed by confocal microscopy. Untreated cells were used as negative control. 


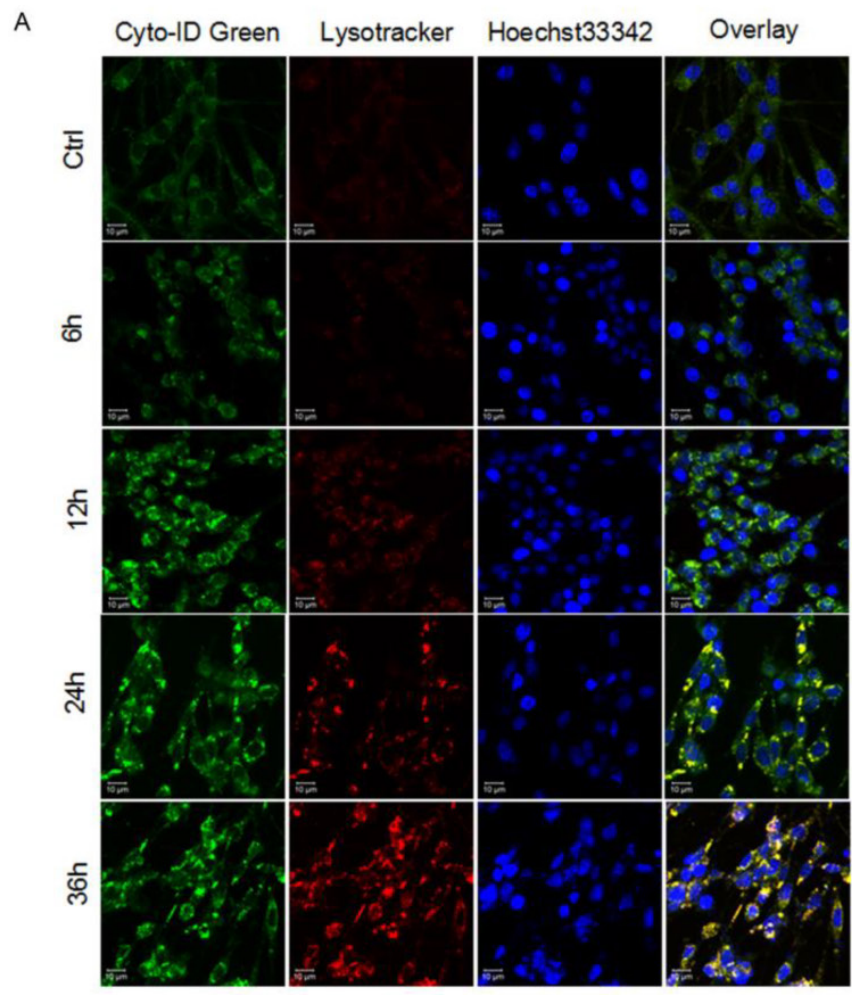

B

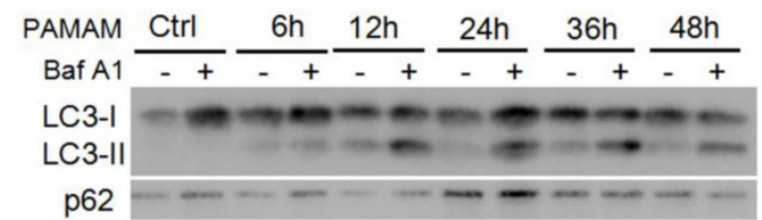

C

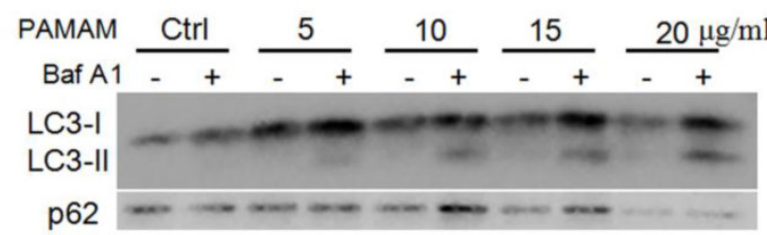

D

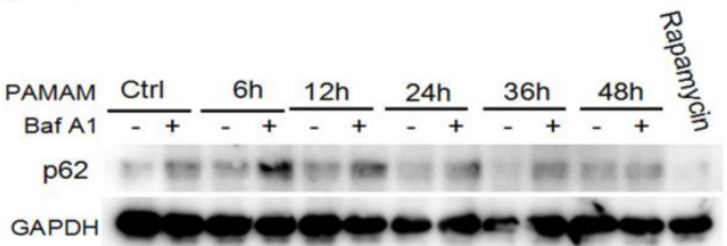

$\mathrm{E}$

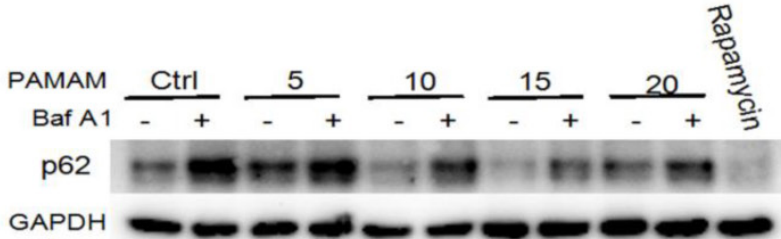

Figure 2. Autophagic flux was induced by PAMAM dendrimers in PC-12 cells. (A) Cells were plated in cell culture dishes with glass bottom. After treated with 10 $\mu \mathrm{g} / \mathrm{mL}$ of PAMAM dendrimers G5 for $6 \mathrm{~h}, 12 \mathrm{~h}, 24 \mathrm{~h}$, and $36 \mathrm{~h}$, cell samples were stained with Cyto-ID Green autophagy dye and Lysotracker Red dye and analyzed by confocal microscopy. Untreated cells were used as negative control. (B) After exposure to $10 \mu \mathrm{g} / \mathrm{mL}$ of PAMAM dendrimers G5 for indicated times, PC-12 cells were treated with or without $20 \mathrm{nM}$ of Baf Al for another $3 \mathrm{~h}$. Changes in the expression of LC3 and p62 were examined by Western blot. (C) After exposure to a series of concentrations of PAMAM dendrimers for $24 \mathrm{~h}, \mathrm{PC}-12$ cells were treated with or without $20 \mathrm{nM}$ of Baf A1 for another $3 \mathrm{~h}$. Changes in expression of LC 3 and p62 were examined by Western blot. (D) After exposure to $10 \mu \mathrm{g} / \mathrm{mL}$ of PAMAM dendrimers G5 for indicated times, PC-12 cells were treated with or without $20 \mathrm{nM}$ of Baf Al for another $3 \mathrm{~h}$. Changes in the expression of p62 was examined by Western blot. Cells treated by $10 \mathrm{nM}$ of rapamycin for $24 \mathrm{~h}$ were as positive control. (E) After exposure to a series of concentrations of PAMAM dendrimers for $24 \mathrm{~h}, \mathrm{PC}-12$ cells were treated with or without $20 \mathrm{nM}$ of Baf Al for another $3 \mathrm{~h}$. Changes in expression of p62 were examined by Western blot. Cells treated by $10 \mathrm{nM}$ of rapamycin for $24 \mathrm{~h}$ were as positive control.

\section{Suppression of reactive oxygen species im- paired PAMAM dendrimers-induced au- tophagic effects in neuronal cells.}

To explore the role of reactive oxygen species in autophagy triggered by PAMAM dendrimers in neurocytes, antioxidants such as NAC, lipoic acid, and tocopherol were applied to inhibit reactive oxygen species in PAMAM dendrimers-treated PC-12 cells. Reactive oxygen species assay kit was used to confirm the suppression of oxidative stress by antioxidants (NAC, lipoic acid, and tocopherol) (Figure 3A). Compared with PAMAM dendrimers treatment alone, pretreatment with antioxidants impaired accumulation of reactive oxygen species-associated fluorescence (red in the web version) induced by PAMAM dendrimers. Meanwhile, autophagic fluorescence (green in the web version) triggered by PAMAM dendrimers was also suppressed by antioxidants (Figure 3B). The larger versions of these confocal images were shown in Figure S8. The quantitative analysis of relative oxidative fluorescence intensity showed that PAMAM dendrimers obviously induced oxidative fluorescence, while antioxidants significantly reduced the accumulation of oxidative fluorescence (Figure 3C). As shown in Figure 3D, antioxidants significantly suppressed the autophagic fluorescence triggered by PAMAM dendrimers in PC-12 cells. Figure S9 showed that antioxidants NAC and lipoic acid could also impair PAMAM dendrimers-induced autophagic fluorescence accumulation in SHSY-5Y cells. Western blot analysis was also used to detect autophagy inhibitory effects of antioxidants. As shown in Figure 3E, antioxidants treatment obviously reduced highly-expressed LC3-II protein level induced by PAMAM dendrimers in PC-12 cells.

In summary, these results suggested that suppression of reactive oxygen species could impair PAMAM dendrimers-induced in PC-12 cells. 
A

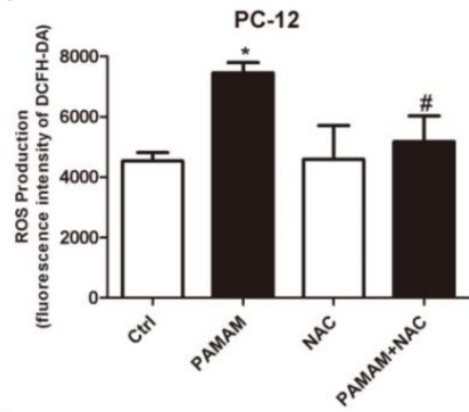

B

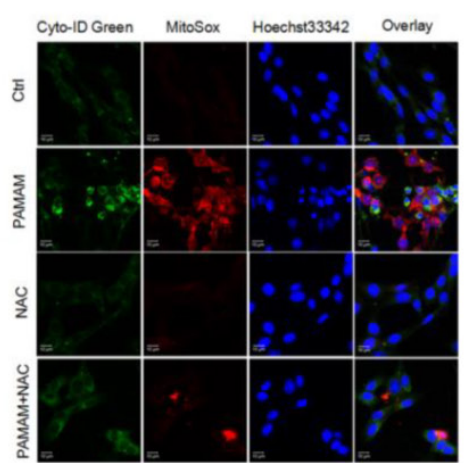

C

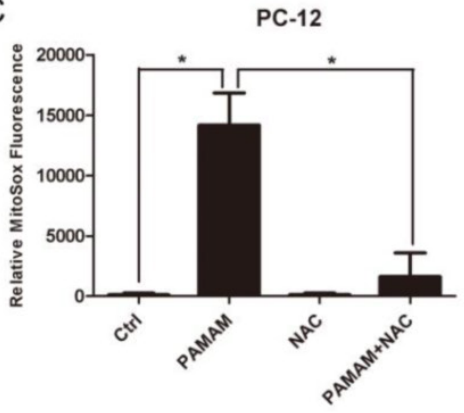

D

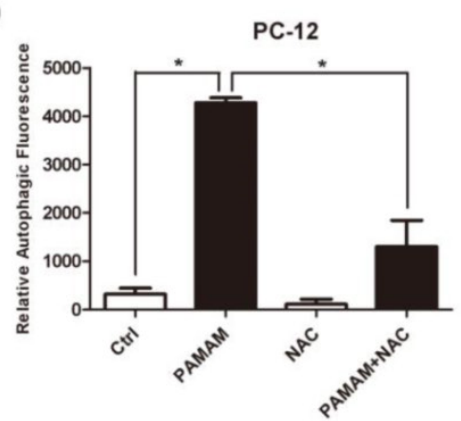

$\mathrm{E}$

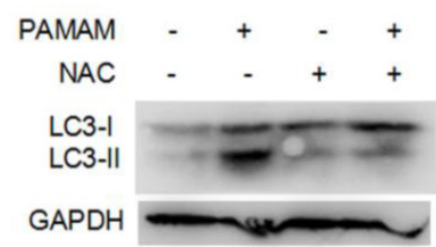

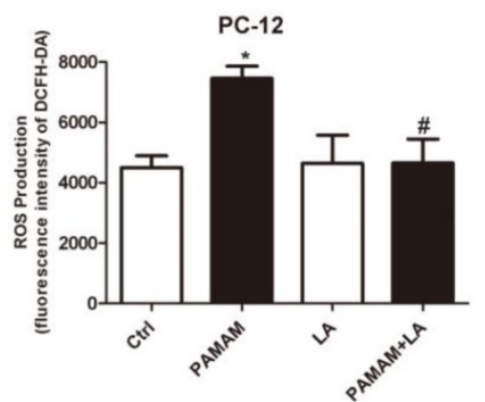
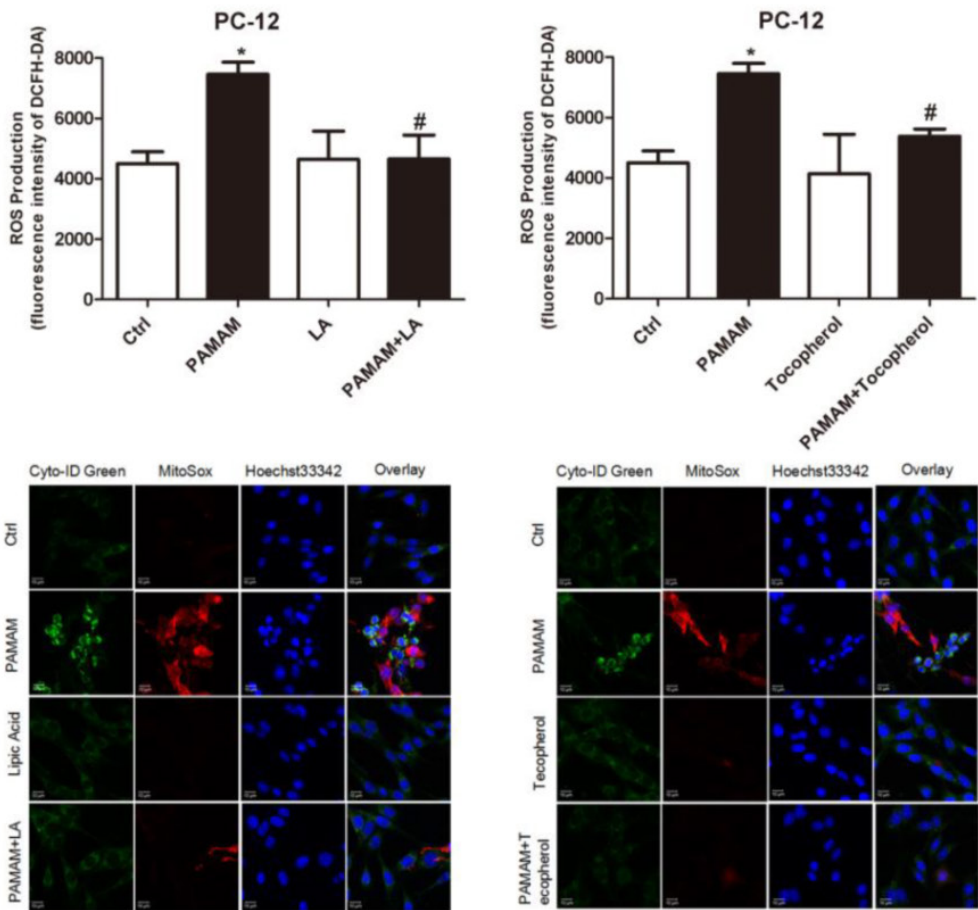

PC-12
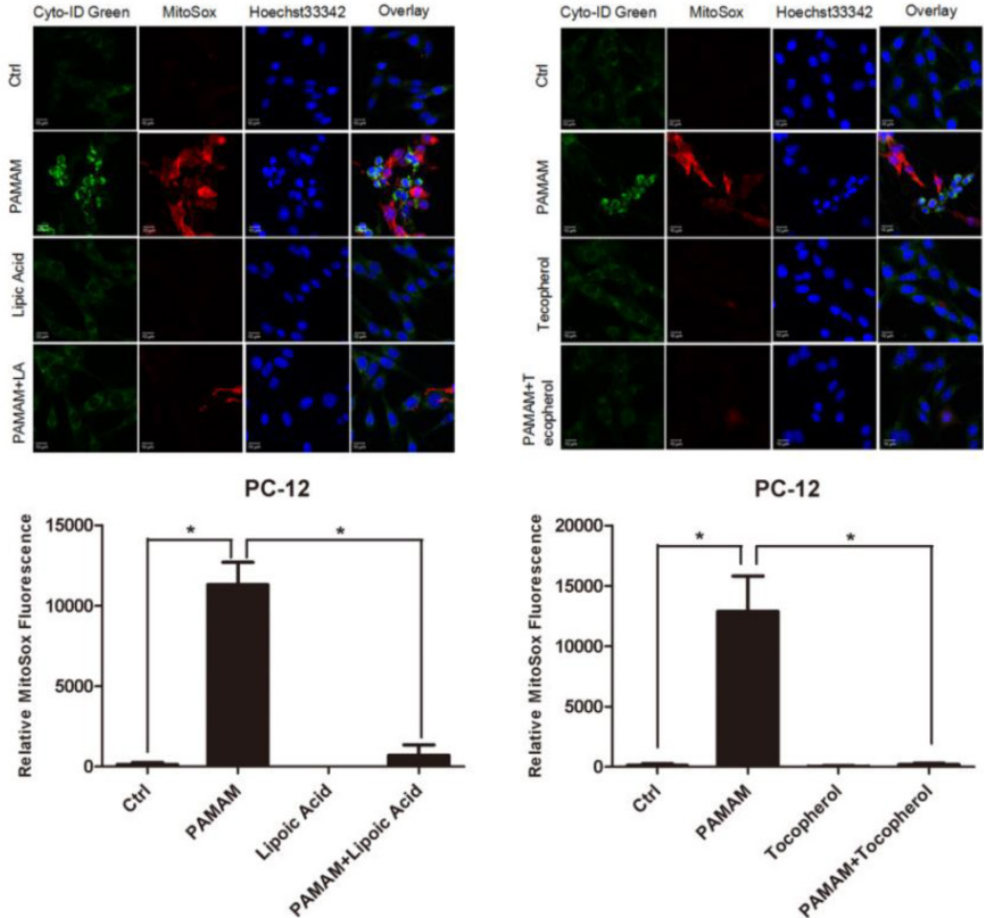

PC-12
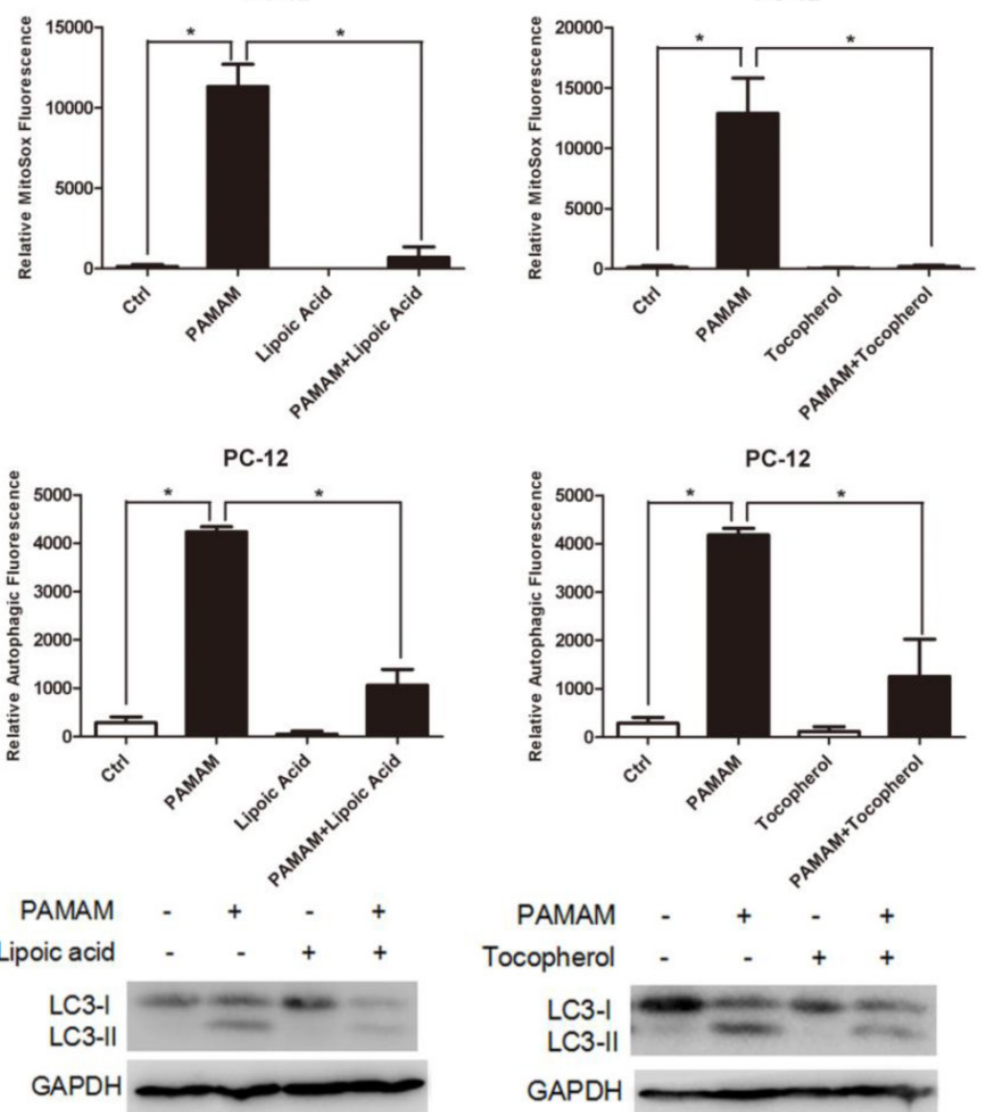

Figure 3. Suppression of reactive oxygen species impaired PAMAM dendrimers-induced autophagic effects. (A) PC-12 cells were incubated with or without PAMAM dendrimers in the presence or absence of antioxidants for $24 \mathrm{~h}$. Cell samples were treated with DCFH-DA and analyzed by microplate reader to quantify levels of ROS. $* p<0.05$ versus $C$ trl, and \#p $<0.05$ versus PAMAM dendrimers $G 5$-treated group. (B) PC- 12 cells were incubated with or without PAMAM dendrimers in the presence or absence of antioxidants for $24 \mathrm{~h}$. Cell samples were stained with Cyto-ID Green dye and MitoSox Red dye and analyzed by confocal microscopy. (C) and (D)The relative oxidative fluorescence and autophagic fluorescence intensity were quantified by confocal microscopy software. All data were presented as the means \pm SD of more than three samples. ${ }^{*} p<0.05$ versus Ctrl, or versus PAMAM dendrimers G5-treated group. (E) PC-12 cells were treated as was described in (A). The whole protein was extracted, and LC 3 was analyzed by Western blot. 


\section{Blocking reactive oxygen species reduced PAMAM dendrimers-induced neuronal cell death.}

To explore whether reactive oxygen species contributed to PAMAM dendrimers-induced neurotoxicity, the growth inhibitory effects induced by PAMAM dendrimers were detected after reactive oxygen species were blocked. As shown in Figure 4A and $4 \mathrm{~B}, \mathrm{PAMAM}$ dendrimers induced growth inhibition of PC-12 cells in a dose-dependent manner, while $5 \mathrm{mM}$ or $10 \mathrm{mM}$ of NAC remarkably impaired cytotoxicity of PAMAM dendrimers. Besides, different

A

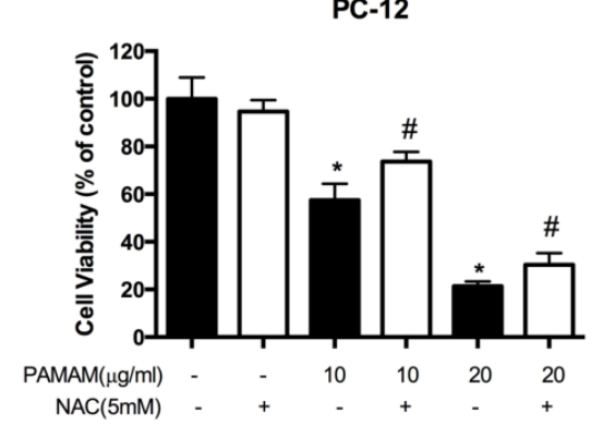

C

PC-12

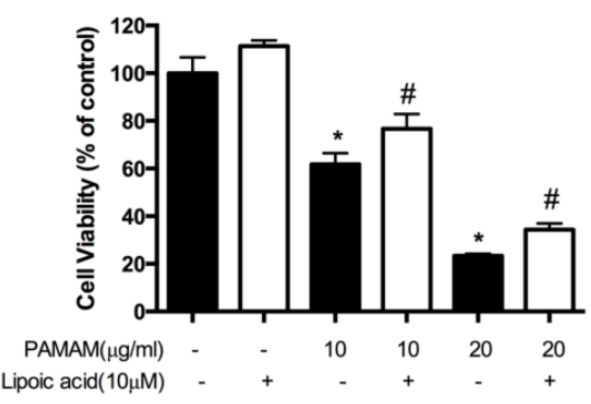

$E$

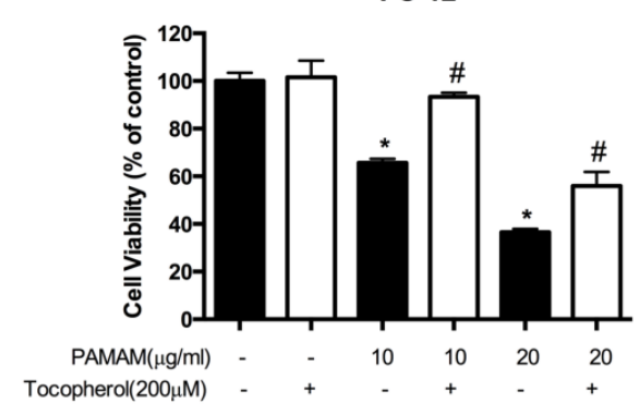

concentrations of antioxidants lipoic acid $(10 \mu \mathrm{M}$ or $100 \mu \mathrm{M})$ and tocopherol $(200 \mu \mathrm{M}$ or $400 \mu \mathrm{M})$ also obviously reduced PAMAM dendrimers-induced neurotoxicity in PC-12 cells (Figure 4C-4F). NAC and lipoic acid were also used to suppress reactive oxygen species accumulated in PAMAM dendrimers-treated human neuroblastoma SHSY-5Y cells, and growth inhibitory effects induced by PAMAM dendrimers were significantly suppressed after adding antioxidants NAC (5 mM and $10 \mathrm{mM})$ or lipoic acid $(10 \mu \mathrm{M}$ and $20 \mu \mathrm{M})$ (Figure S10).

B

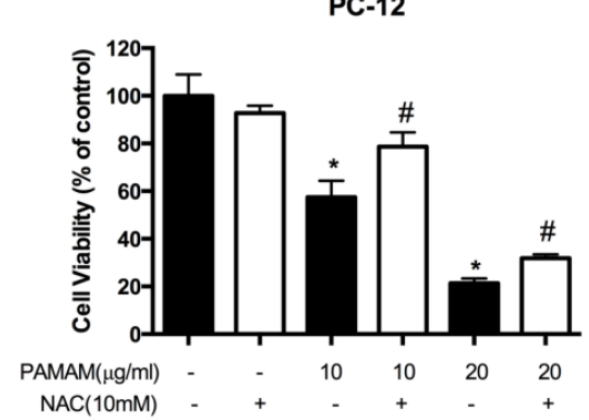

D

PC-12

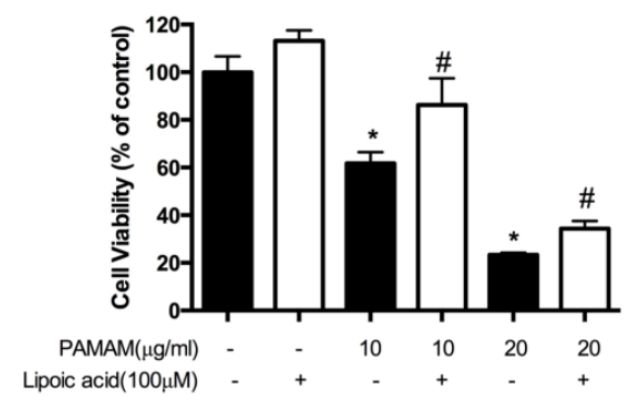

F

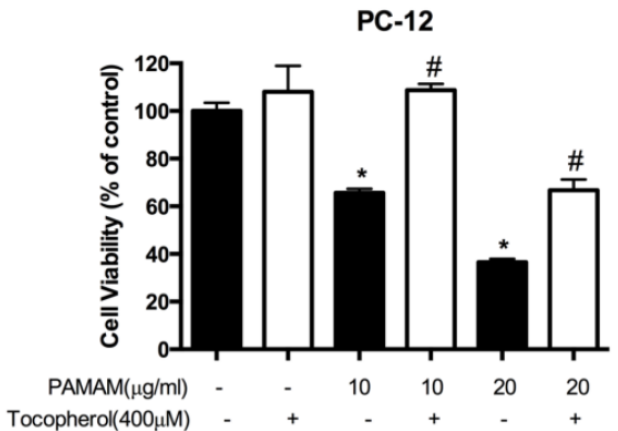

Figure 4. Blocking reactive oxygen species reduced PAMAM dendrimers-induced neuronal cell death. (A) and (B) PC-12 cells were incubated with or without PAMAM dendrimers in the presence or absence of NAC for $24 \mathrm{~h}$. Cell growth inhibition was analyzed by CCK-8. All data were presented as the means \pm SD of four samples. $* p<0.05$ versus $C$ trl, and \#p $<0.05$ versus PAMAM dendrimers G5-treated group. (C) and (D) PC-12 cells were incubated with or without PAMAM dendrimers in the presence or absence of lipic acid for $24 \mathrm{~h}$. Cell growth inhibition was analyzed by CCK-8. All data were presented as the means \pm SD of four samples. $* p<0.05$ versus Ctrl, and \#p<0.05 versus PAMAM dendrimers G5-treated group. (E) and (F) PC-12 cells were incubated with or without PAMAM dendrimers in the presence or absence of tocopherol for $24 \mathrm{~h}$. Cell growth inhibition was analyzed by CCK- 8 assay. All data were presented as the means \pm SD of four samples. * $p<0.05$ versus Ctrl, and $\# p<0.05$ versus PAMAM dendrimers G5-treated group. 


\section{Inhibition of autophagy protected against PAMAM dendrimers-induced neuronal cell death.}

To assess the role of autophagy in PAMAM dendrimers-induced neuronal cell death, two autophagy inhibitors, LY294002 and CQ, were used to inhibit PAMAM dendrimers-induced autophagy. LY294002 is an inhibitor of phosphatidylinositol 3-phosphate kinase (PI3K) which could reduce the protein level of autophagosomic LC3 (LC3-II), while CQ inhibits fusion between the autophagosomes and lysosomes, and elevates LC3-II[38, 39]. Confocal microscopy and Western blot analyses showed that LY294002 inhibited autophagy induced by PAMAM dendrimers in PC-12 cells and decreased LC3-II formation (Figure 5A and 5C). In contrast, PAMAM dendrimers in combination with $\mathrm{CQ}$ enhanced green fluorescence and LC3-II protein levels (Figure 5E and $5 \mathrm{G})$. The quantitative analysis of relative autophagic fluorescence intensity showed that LY294002 significantly impaired PAMAM dendrimers-triggered autophagic fluorescence, while CQ treatment enhanced autophagic fluorescence (Figure 5B and 5F). Moreover, the pre-processing of autophagy inhibitors (LY294002 or CQ) before PAMAM dendrimers exposure significantly reduced the cytotoxicity of PAMAM dendrimers in PC-12 cells (Figure 5D and 5H).

To further confirm the role of autophagy induced by PAMAM dendrimers in PC-12 cells, two autophagy activators, trehalose and rapamycin, were employed to detect the effects of autophagy activation on the neurotoxicity of PAMAM dendrimers[40, 41]. Confocal microscopy and western blot analysis showed that trehalose and rapamycin clearly enhanced autophagy induced by PAMAM dendrimers in PC-12 cells by inducing autophagic fluorescence accumulation (Figure 6A and 6E) and LC3-II protein formation (Figure 6C and 6G). The quantitative analyses of relative autophagic fluorescence intensity were shown in figure $6 \mathrm{~B}$ and $6 \mathrm{~F}$. Compared with PAMAM dendrimers treatment alone, trehalose and rapamycin significantly enhanced the toxicity of PAMAM dendrimers in PC-12 cells (Figure 6D and $6 \mathrm{H})$.

Taken together, these results confirmed that inhibition of autophagy protected against PAMAM dendrimers-induced neuronal cell death.

\section{AKT/MTOR signaling pathways were involved in PAMAM dendrimers-induced autophagy.}

AKT/MTOR/p70S6K signaling pathway plays an important role in cell proliferation, survival and apoptosis, and is one of the primary pathways regulating autophagy in eukaryotic cells[42]. Researchers have demonstrated that autophagy is negatively reg- ulated by the activation of the mammalian target of rapamycin (MTOR) as MTORC1 could regulate autophagy in response to pathophysiological stress[43, 44]. As showed in Figure 7A and 7B, PAMAM dendrimers decreased the phosphorylation (at Ser473) of the AKT protein in PC-12 cells in a dose- and time-dependent manner, respectively. The change of phosphorylated form of the MTOR (at Ser2448) after PC-12 cells were exposed to PAMAM dendrimers was similar to that of phosphorylated AKT protein. Exposure to PAMAM dendrimers also resulted in a diminished level of phosphorylation of the MTOR target, p70 ribosomal protein S6 kinase (p70S6K), revealing a potent inhibitory effect of the PAMAM dendrimers treatment on the AKT/MTOR signaling pathway.

\section{ERK 1/2 signaling pathway was activated in oxidative stress and autophagy induced by PAMAM dendrimers.}

The mitogen-activated protein kinases (MAPKs) involving extracellular signal-regulated kinase (ERK), JNK and p38, have been found to be the protein responsible for ROS induction[11]. Meanwhile, MAPK activation has been previously reported to contribute to autophagy effects[45]. Thus, ERK $1 / 2$ signaling pathway may play an important role in oxidative stress and autophagy induced by PAMAM dendrimers.

As shown in Figure 8A, exposure of PC-12 cells to PAMAM dendrimers resulted in an increase of ERK $1 / 2$ phosphorylation (at T202/Y204) in a doseand time- dependent manner. To further investigate the role of ERK 1/2 in oxidative stress and autophagy induced by PAMAM dendrimers, an inhibitor of MEK1 and MEK2, U0126, was used to block the phosphorylation of ERK 1/2. PC-12 cells were incubated with $20 \mu \mathrm{M}$ of U0126 for $2 \mathrm{~h}$, and then treated with PAMAM dendrimers for $24 \mathrm{~h}$. Reactive oxygen species assay showed that U0126 could obviously suppress the generation of reactive oxygen species triggered by PAMAM dendrimers in PC-12 cells (Figure 8B). Confocal microscopy analyses presented that inhibiting phosphorylated ERK 1/2 notably weakened autophagic fluorescence and oxidative fluorescence signal in PAMAM dendrimers-treated PC-12 cells (Figure 8C). The quantitative analysis of relative fluorescence intensity showed that U0126 significantly impaired both autophagic fluorescence and oxidative fluorescence induced by PAMAM dendrimers in PC-12 cells (Figure 8D and 8E). Meanwhile, western blot analysis showed that phosphorylation of ERK 1/2 was suppressed by U0126, and inhibition of phosphorylation of ERK 1/2 obviously decreased the protein level of LC3-II (Figure 
8F). Furthermore, suppression of ERK 1/2 signaling pathway could significantly protect against PAMAM

\section{A}
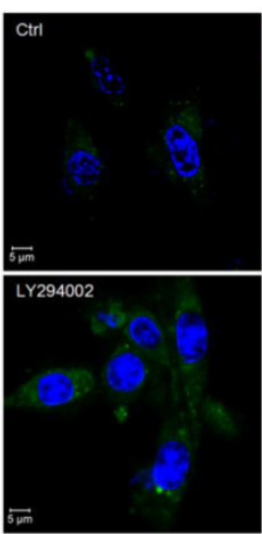

B

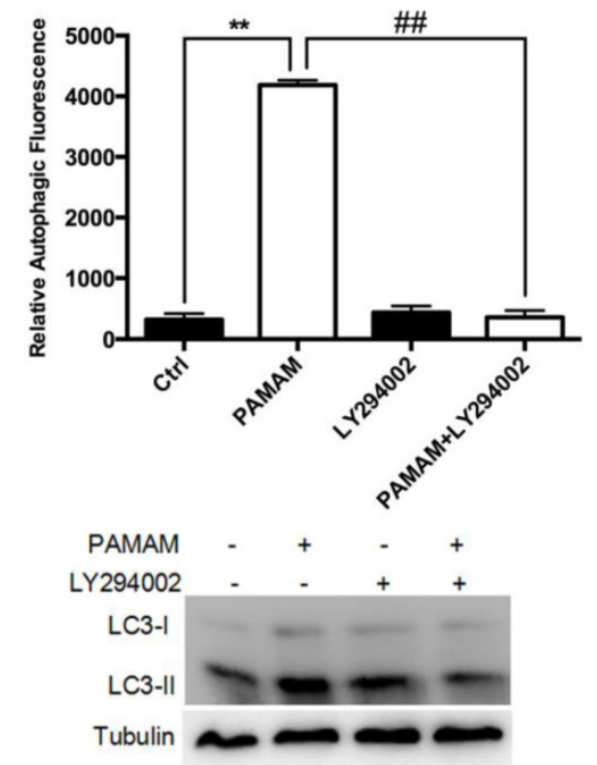

PC-12

D

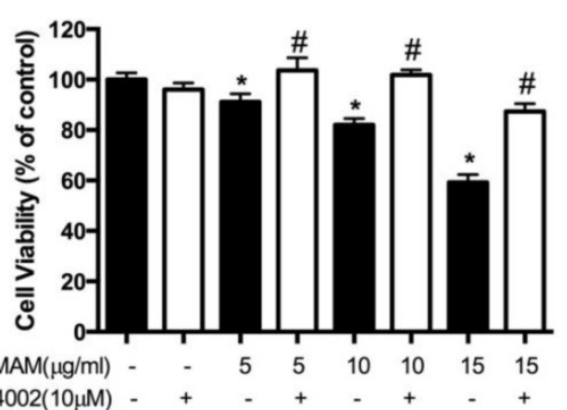

dendrimers-induced neurotoxicity in PC-12 cells (Figure 8G).

$\mathrm{E}$

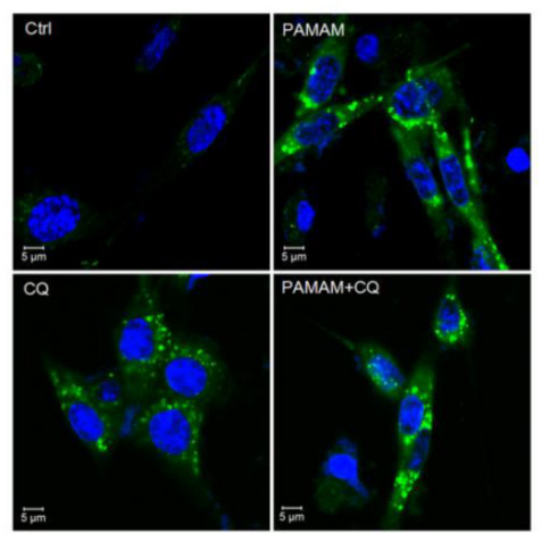

$\mathrm{F}$

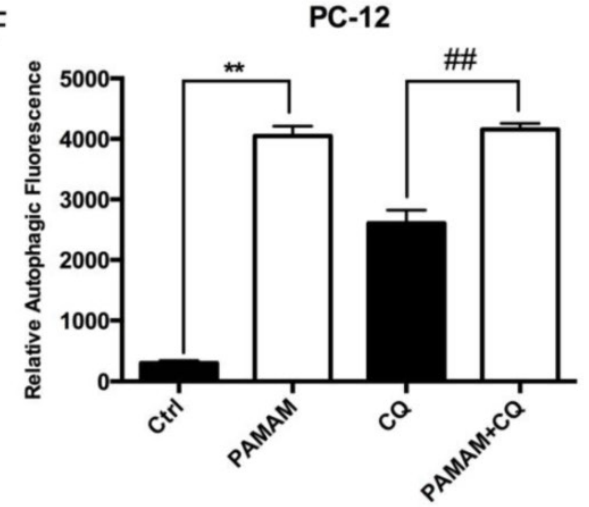

G

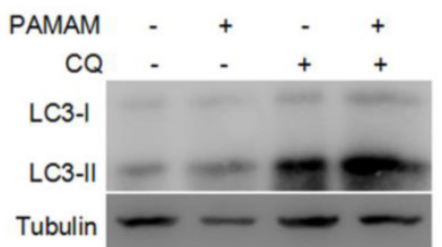

PC-12

$\mathrm{H}$

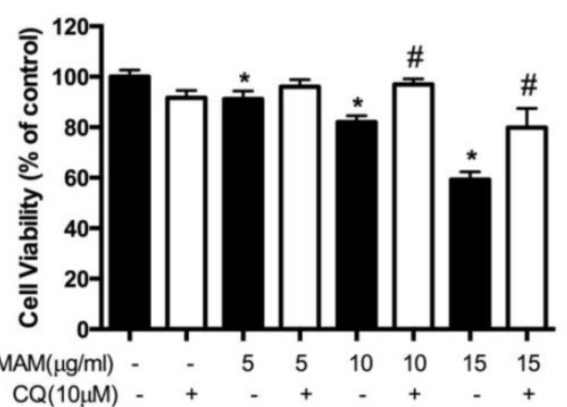

Figure 5. Inhibition of autophagy protected against PAMAM dendrimers-induced neuronal cell death. (A) PC-12 cells were incubated with or without PAMAM dendrimers in the presence or absence of the autophagy inhibitor LY294002 for $24 \mathrm{~h}$. Cell samples were stained with Cyto-ID Green dye and analyzed by confocal microscopy. Untreated cells were used as negative control. (B) The relative autophagic fluorescence intensity was quantified by confocal microscopy software. All data were presented as the means \pm SD of more than three samples. (C) PC-12 cells were treated as described in (A). The whole protein was extracted, and LC3 was analyzed by Western blot. (D) PC-12 cells were treated as was described in (A). Cell growth inhibition was analyzed by CCK-8. All data were presented as the means \pm SD of four samples. $* p<0.05$ versus $C$ trl, and $\# p<0.05$ versus PAMAM dendrimers G5-treated group. (E) PC-12 cells were incubated with or without PAMAM dendrimers in the presence or absence of the autophagy inhibitor CQ for $24 \mathrm{~h}$. Cell samples were stained with Cyto-ID Green autophagy dye and analyzed by confocal microscopy. Untreated cells were used as negative control. (F) The relative autophagic fluorescence intensity was quantified by confocal microscopy software. All data were presented as the means \pm SD of more than three samples. (G) $P C-12$ cells were treated as was described in (E). The whole protein was extracted, and LC3 was analyzed by Western blot. (H) PC-12 cells were treated as was described in (E). Cell growth inhibition was analyzed by CCK-8. All data were presented as the means \pm SD of four samples. * $p<0.05$ versus Ctrl, and \#p $<0.05$ versus PAMAM dendrimers G5-treated group. 
A
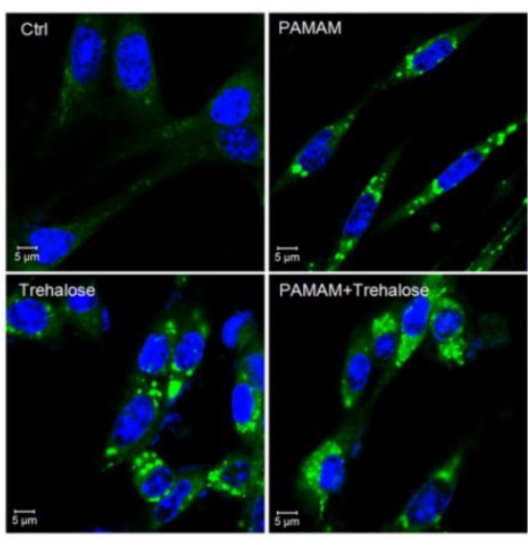

B

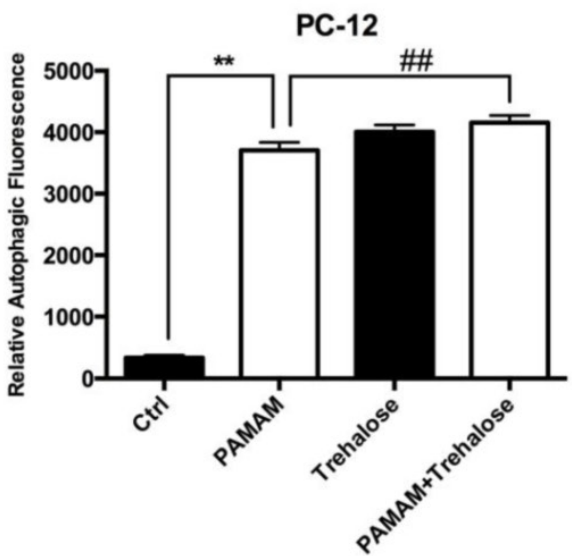

C

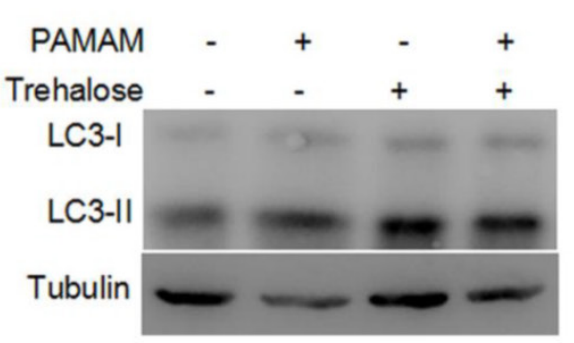

D

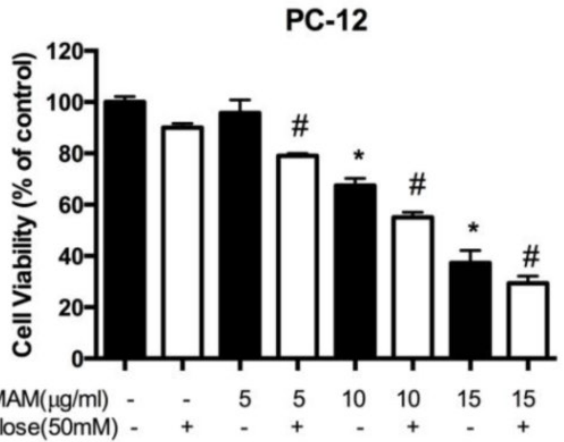

E

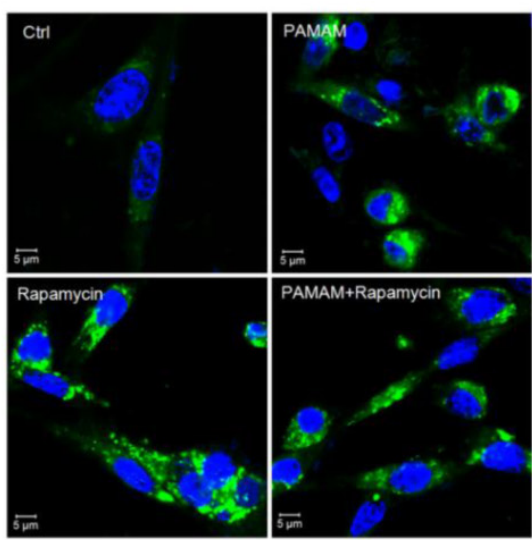

$\mathrm{F}$

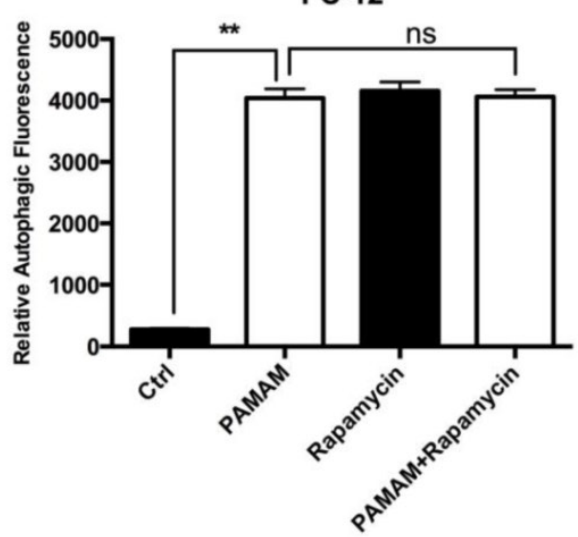

G

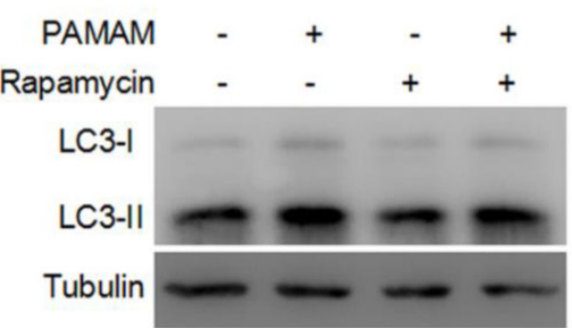

$\mathrm{H}$

PC-12

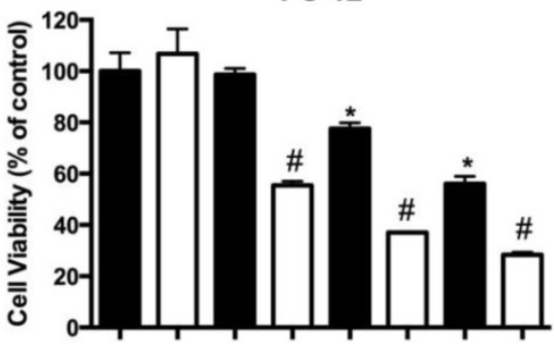

$\operatorname{PAMAM}(\mu \mathrm{g} / \mathrm{ml}) \quad-\quad-\quad \begin{array}{llllll}5 & 5 & 10 & 10 & 15 & 15\end{array}$

Rapamycin(10nM) - + - + + - +

Figure 6. Autophagy activators enhanced cytotoxicity of PAMAM dendrimers in PC-12 cells. (A) PC-12 cells were incubated with or without PAMAM dendrimers in the presence or absence of the autophagy inducers trehalose for $24 \mathrm{~h}$. Cell samples were stained with Cyto-ID Green autophagy dye and analyzed by confocal microscopy. Untreated cells were used as negative control. (B) The relative autophagic fluorescence intensity was quantified by confocal microscopy software. All data were presented as the means \pm SD of more than three samples. (C) PC- 12 cells were treated as was described in (A). The whole protein was extracted, and LC 3 was analyzed by Western blot. (D) PC-12 cells were treated as was described in (A). Cell growth inhibition was analyzed by CCK-8. All data were presented as the means \pm SD of four samples. * $p<0.05$ versus Ctrl, and $\# p<0.05$ versus PAMAM dendrimers G5-treated group. (E) PC-12 cells were incubated with or without PAMAM dendrimers in the presence or absence of the autophagy inducer rapamycin for $24 \mathrm{~h}$. Cell samples were stained with Cyto-ID Green dye and analyzed by confocal microscopy. Untreated cells were used as negative control. ( $F$ ) The relative autophagic fluorescence intensity was quantified by confocal microscopy software. All data were presented as the means \pm SD of more than three samples. (G) $P C-12$ cells were treated as was described in (E). The whole protein was extracted, and LC3 was analyzed by Western blot. (H) PC-12 cells were treated as was described in (E). Cell growth inhibition was analyzed by CCK-8 assay. All data were presented as the means \pm SD of four samples. $* p<0.05$ versus Ctrl, and \#p<0.05 versus PAMAM dendrimers G5-treated group. 
A

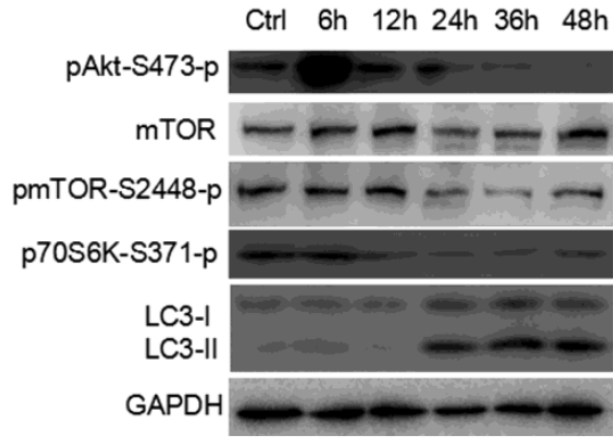

B

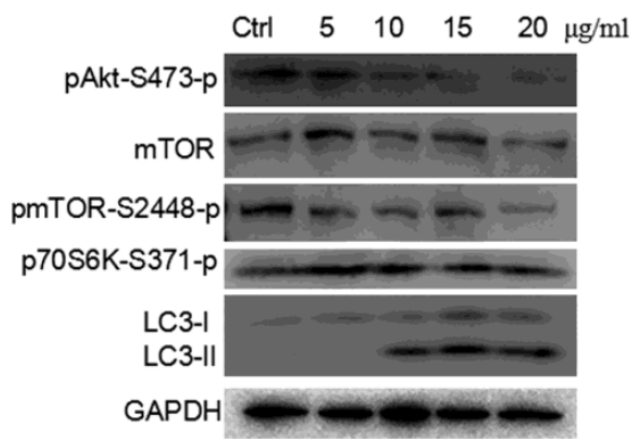

Figure 7. AKT/MTOR signaling pathways were involved in PAMAM dendrimers-induced autophagy. (A) PC-12 cells were seeded in a 6-well plate at an initial density of $2 \times 10^{5}$ cells per well. After $24 \mathrm{~h}$ of stabilization, cells were treated with $10 \mu \mathrm{g} / \mathrm{mL}$ of PAMAM dendrimers $\mathrm{G} 5$ for indicated times. The same blot was used to probe pAKT, mTOR, pMTOR, pP70S6K, and LC3. Changes in pAKT, mTOR, pMTOR, pP70S6K, and LC3 were examined by Western blot. (B) PC-12 cells were treated with different concentrations of PAMAM dendrimers G5 for $24 \mathrm{~h}$. The same blot was used to probe pAKT, mTOR, pMTOR, pP70S6K, and LC3. Changes in the expression of pAKT, mTOR, PMTOR, PP70S6K, and LC3 were examined by Western blot.

Collectively, our results suggested that ERK $1 / 2$ signaling pathway played a key role in interplay between oxidative stress and autophagy induced by PAMAM dendrimers in PC-12 cells.

\section{Discussion}

Up to date, it has been reported that cationic PAMAM dendrimers could induce acute lung injury and liver damage, disrupt key platelet functions, initiate blood clot formation, impair mitochondrial oxidation in brain tissue, and induce central nervous system injury[7-9]. Meanwhile, PAMAM dendrimers-induced cytotoxicity was tightly correlated with the physical properties of nanomaterials themselves, such as surface charge and generation[46, 47], but the understanding of underlying interaction mechanism between PAMAM dendrimers and organ during their toxicity was still limited. Several literatures have reported that PAMAM dendrimers could induce nanoscale holes formation, trigger net cell plasma membrane porosity, and activate lysosomal apoptotic pathway at therapeutically relevant concentrations[48]. Meanwhile, PAMAM dendrimers could induce fibrinogen aggregation and contribute to disseminate intravascular coagulation (DIC)-like condition through electrostatic interaction between cationic dendrimer surface and negatively charge fibrinogen domain [9].

In terms of mechanisms of toxicity, researchers have demonstrated that the accumulation of reactive oxygen species was the main mechanism of PAMAM dendrimers-induced toxicity[32]. Meanwhile, our previous studies have pointed out that autophagy, an evolutionarily conserved process for engulfing cytoplasmic materials into autophagosomes and degrading damaged organelles in lysosomes may represent a new mechanism in toxicity triggered by PAMAM dendrimers[24, 29, 49]. Nowadays, nanomaterials such as quantum dots, silver nanoparticles, zinc ox- ide, and titanium dioxide nanoparticles, have been widely proved to induce autophagy and oxidative stress in various cells. Oxidative stress and autophagy could be triggered independently or dependently of each other in nanoparticles-induced toxicity: Lee ever reported cytotoxicity, oxidative stress, apoptosis and the autophagic effects of silver nanoparticles in mouse embryonic fibroblasts independently[13]; while Wan reported that gold nanorods could induce cell apoptosis and autophagy by damaging mitochondria and activating intracellular reactive oxygen species[50], Gorojod pointed out that manganese-mediated generation of reactive oxygen species could promote cellular damage, leading to apoptotic cell death in neuronal cells, while the autophagic-lysosomal pathway could determine the fate of neuronal cells under manganese-induced oxidative stress condition[51]. Thus, the relation between reactive oxygen species and autophagy in nanoparticles-triggered toxicity is confusing, and whether reactive oxygen species were essential for autophagy induction is still unclear, especially in PAMAM dendrimers-induced neurotoxicity. To address this problem, the effects of a series of antioxidants on autophagy and cytotoxicity in PAMAM dendrimers-treated neuronal cells were investigated. Incubation with antioxidants before PAMAM dendrimers exposure could obviously impair autophagic effects, abolish the formation of LC3-II and reduce cytotoxicity in neuronal cells. Meanwhile, autophagy inhibitors could also protect against PAMAM dendrimers-induced neuronal cell death, whereas autophagy activators could enhance PAMAM dendrimers-induced growth inhibitory effect in PC-12 cells. These results in the present study ascertained the capacity of reactive oxygen species triggered by PAMAM dendrimers to induce autophagy in neuronal cells at an early stage, and revealed that antioxidants or autophagy inhibitors could impair PAMAM dendrimers-induced neurotoxicity. 
A

Ctrl $6 \mathrm{~h} 12 \mathrm{~h} 24 \mathrm{~h} 36 \mathrm{~h} \quad 48 \mathrm{~h}$

Erk1/2

Erk1/2-T202/Y204-p

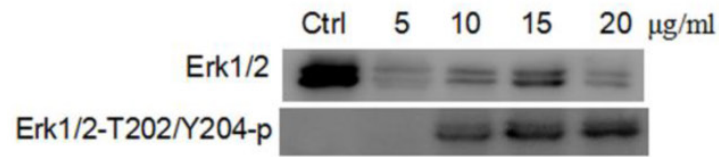

C

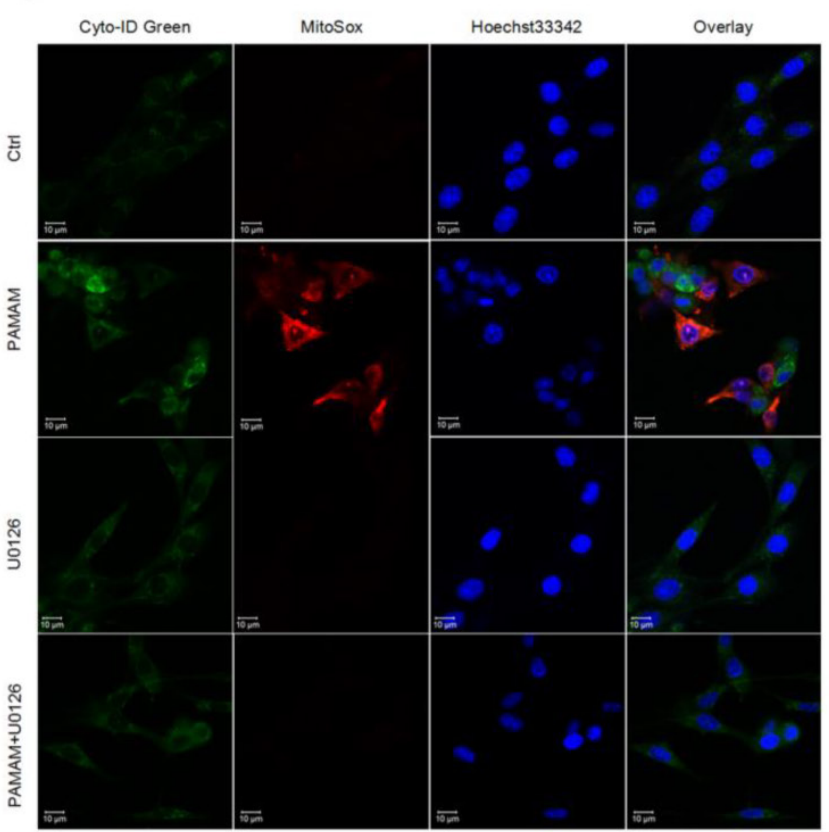

$\mathrm{F}$

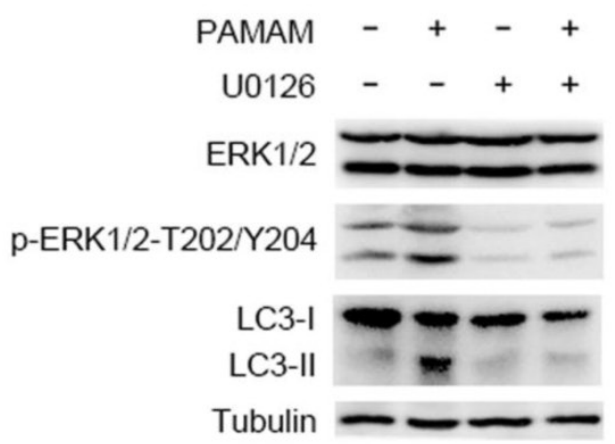

B

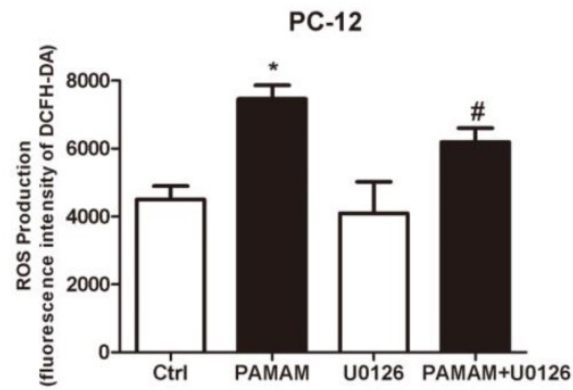

D

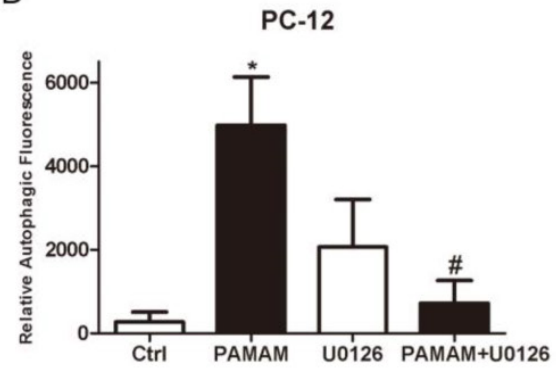

E

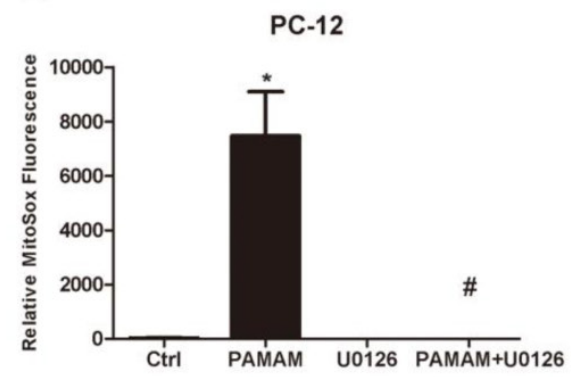

G

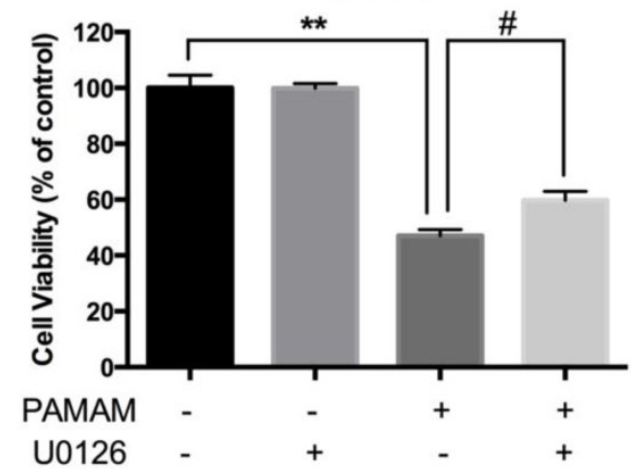

Figure 8. ERK $1 / 2$ signaling pathway was activated in oxidative stress and autophagy induced by PAMAM dendrimers. (A) PC-12 cells were seeded in a 6-well plate at an initial density of $2 \times 10^{5}$ cells per well. After $24 \mathrm{~h}$ of stabilization, cells were treated with $10 \mu \mathrm{g} / \mathrm{mL}$ of PAMAM dendrimers G5 for indicated times, or treated with different concentrations of PAMAM dendrimers G5 for $24 \mathrm{~h}$. The same blot was used to probe Erk $1 / 2$ and $\mathrm{pErk} 1 / 2$. Changes in Erk $1 / 2$ and $p$ Erk $1 / 2$ were examined by Western blot. (B) PC-12 cells were incubated with or without PAMAM dendrimers in the presence or absence of U0126 for 24h. Cell samples were treated with DCFH-DA and analyzed by microplate reader to quantify levels of ROS. $* p<0.05$ versus Ctrl, and $\# p<0.05$ versus PAMAM dendrimers G5-treated group. (C) PC-12 cells were treated as was described in (B). Cell samples were stained with Cyto-ID Green autophagy dye and MitoSox Red dye and analyzed by confocal microscopy. (D) and (E) The relative oxidative fluorescence intensity was quantified by confocal microscopy software. All data were presented as the means \pm SD of more than three samples. ${ }^{*}<0.05$ versus Ctrl, or versus PAMAM dendrimers G5-treated group. $(F)$ Cells were treated as was described in (B), the whole protein was extracted, changes in Erk 1/2, pErk 1/2, and LC 3 were analyzed by Western blot. (G) Cell growth inhibition was analyzed by CCK-8 assay. All data were presented as the means \pm SD of four samples. $* p<0.05$ versus Ctrl, and \#p<0.05 versus PAMAM dendrimers G5-treated group.

To further explore the underlying mechanism between oxidative stress and autophagy in PAMAM dendrimers-treated PC-12 cells, the activation of ERK
$1 / 2$ signaling pathway was examined. Recent studies have confirmed that ERK 1/2 was tightly responsible for ROS induction: MEK inhibitor could protect cells 
against oxidative stress[52]; furthermore, our previous study found that ERK $1 / 2$ activation contributed to autophagic effects[30]; Autophagy proteins regulate ERK phosphorylation, and direct ERK activation promotes autophagy even in the absence of other stimuli[53, 54]. In this study, U0126, inhibitor of MEK1 and MEK2, was used to block the phosphorylation of ERK $1 / 2$ triggered by PAMAM dendrimers. Oxidative effect and autophagic effect were both impaired after inhibiting ERK 1/2 phosphorylation. Meanwhile, the growth inhibitory effect induced by PAMAM dendrimers was also suppressed, which is similar to the effects of antioxidants or autophagy inhibitors, suggesting that ERK $1 / 2$ signaling pathway might directly contribute to the interplay between oxidative stress and autophagy in PAMAM dendrimers-treated PC-12 cells.

\section{Conclusion}

We demonstrated that reactive oxygen species and autophagic flux were triggered by PAMAM dendrimers in neuronal cells. Meanwhile, suppression of reactive oxygen species impaired autophagy and cytotoxicity induced by PAMAM dendrimers. Notably, inhibition of autophagy significantly protected against PAMAM dendrimers-induced neuronal cell death. These results indicated that PAMAM dendrimers-induced autophagy might be triggered by reactive oxygen species in neurotoxic response of PAMAM dendrimers, and antioxidants or autophagy inhibitors could be used to mitigate PAMAM dendrimers-induced neurotoxicity, thus providing potential strategies for alleviating the neurotoxicity of PAMAM dendrimers. Furthermore, the systematical elucidation of interrelation between reactive oxygen species and autophagy will also encourage the development of safe biomedical nanomaterials.

\section{Supplementary Material}

Supplemental Figures 1-11.

http://www.thno.org/v05p1363s1.pdf

\section{Abbreviations}

PAMAM dendrimers: Poly-amidoamine dendrimers; ROS: reactive oxygen species; NAC: N-acetyl cysteine; LA: lipoic acid; CQ: chloroquine; HRP: horseradish peroxidase; IgG: immunoglobulin G; PBS: phosphate-buffered saline; CCK-8: Cell Counting Kit-8; BCA: bicinchoninic acid; SDS-PAGE: sodium dodecyl sulfate-polyacrylaminde gelelectrophoresis; PVDF: poly vinylidene fluoride membranes; BSA: bovine serum albumin; DCFH-DA: 2',7'-dichlorofluorescein diacetate; SD: standard deviations; PI3K: phosphatidylinositol 3-phosphate kinase; MTOR: the mammalian target of rapamycin;
p70S6K: p70 ribosomal protein S6 kinase; MAPKs: mitogen-activated protein kinases; ERK: extracellular signal-regulated kinase.

\section{Acknowledgement}

This work is supported by grants from the National Key Basic Research Program of China (2013CB932502, 2015CB931800), the National Natural Science Foundation of China (81573332), and Shanghai Science and Technology Funds (14431900200).

\section{Competing Interests}

The authors have declared that no competing interest exists.

\section{References}

1. Zhang G, Zeng X, Li P. Nanomaterials in cancer-therapy drug delivery system. J Biomed Nanotechnolo. 2013; 9: 741-50.

2. Mukerjee A, Ranjan AP, Vishwanatha JK. Combinatorial nanoparticles for cancer diagnosis and therapy. Curr Med Chem. 2012; 19: 3714-21.

3. He H, Li Y, Jia XR, Du J, Ying X, Lu WL, et al. PEGylated Poly(amidoamine) dendrimer-based dual-targeting carrier for treating brain tumors. Biomaterials. 2011; 32: 478-87.

4. Bronich T. Multifunctional polymeric carriers for gene and drug delivery. Pharm Res. 2010; 27: 2257-9.

5. Pan B, Cui D, Sheng Y, Ozkan C, Gao F, He R, et al. Dendrimer-modified magnetic nanoparticles enhance efficiency of gene delivery system. Cancer Res. 2007; 67: 8156-63.

6. Duncan R, Izzo L. Dendrimer biocompatibility and toxicity. Adv Drug Deliv Rev. 2005; 57: 2215-37.

7. Li C, Liu H, Sun Y, Wang H, Guo F, Rao S, et al. PAMAM nanoparticles promote acute lung injury by inducing autophagic cell death through the Akt-TSC2-mTOR signaling pathway. J Mol Cell Biol. 2009; 1: 37-45.

8. Jones CF, Campbell RA, Brooks AE, Assemi S, Tadjiki S, Thiagarajan G, et al. Cationic PAMAM dendrimers aggressively initiate blood clot formation. ACS nano. 2012; 6: 9900-10.

9. Jones CF, Campbell RA, Franks Z, Gibson CC, Thiagarajan G, Vieira-de-Abreu A, et al. Cationic PAMAM dendrimers disrupt key platelet functions. Mol Pharm. 2012; 9: 1599-611.

10. Albertazzi L, Gherardini L, Brondi M, Sulis Sato S, Bifone A, Pizzorusso T, et al. In vivo distribution and toxicity of PAMAM dendrimers in the central nervous system depend on their surface chemistry. Mol Pharm. 2013; 10: 249-60.

11. Fu PP, Xia Q, Hwang HM, Ray PC, Yu H. Mechanisms of nanotoxicity: generation of reactive oxygen species. J Food Drug Anal. 2014; 22: 64-75.

12. Manke A, Wang L, Rojanasakul Y. Mechanisms of nanoparticle-induced oxidative stress and toxicity. Biomed Res Int. 2013; 2013: 942916.

13. Lee YH, Cheng FY, Chiu HW, Tsai JC, Fang CY, Chen CW, et al. Cytotoxicity, oxidative stress, apoptosis and the autophagic effects of silver nanoparticles in mouse embryonic fibroblasts. Biomaterials. 2014; 35: 4706-15.

14. Huerta-Garcia E, Perez-Arizti JA, Marquez-Ramirez SG, Delgado-Buenrostro NL, Chirino YI, Iglesias GG, et al. Titanium dioxide nanoparticles induce strong oxidative stress and mitochondrial damage in glial cells. Free Radic Biol Med. 2014; 73: 84-94.

15. Roy R, Singh SK, Chauhan LK, Das M, Tripathi A, Dwivedi PD. Zinc oxide nanoparticles induce apoptosis by enhancement of autophagy via PI3K/Akt/mTOR inhibition. Toxicol Lett. 2014; 227: 29-40.

16. Naha PC, Davoren M, Lyng FM, Byrne HJ. Reactive oxygen species (ROS) induced cytokine production and cytotoxicity of PAMAM dendrimers in J774A.1 cells. Toxicol Appl Pharmacol. 2010; 246: 91-9.

17. Wang $\mathrm{W}$, Xiong $\mathrm{W}$, Wan J, Sun $\mathrm{X}, \mathrm{Xu} \mathrm{H}$, Yang $\mathrm{X}$. The decrease of PAMAM dendrimer-induced cytotoxicity by PEGylation via attenuation of oxidative stress. Nanotechnology. 2009; 20: 105103.

18. Levine B, Kroemer G. Autophagy in the pathogenesis of disease. Cell. 2008; 132: 27-42.

19. Mizushima N. Autophagy: process and function. Genes Dev. 2007; 21: 2861-73.

20. Jain MV, Paczulla AM, Klonisch T, Dimgba FN, Rao SB, Roberg K, et al. Interconnections between apoptotic, autophagic and necrotic pathways: implications for cancer therapy development. I Cell Mol Med. 2013; 17: 12-29.

21. Ha SW, Weitzmann MN, Beck GR, Jr. Bioactive silica nanoparticles promote osteoblast differentiation through stimulation of autophagy and direct association with LC3 and p62. ACS nano. 2014; 8: 5898-910.

22. Song W, Soo Lee S, Savini M, Popp L, Colvin VL, Segatori L. Ceria nanoparticles stabilized by organic surface coatings activate the lysosome-autophagy system and enhance autophagic clearance. ACS nano. 2014; 8: 10328-42. 
23. Chen L, Miao Y, Chen L, Jin P, Zha Y, Chai Y, et al. The role of elevated autophagy on the synaptic plasticity impairment caused by CdSe/ZnS quantum dots. Biomaterials. 2013; 34: 10172-81.

24. Wang S, Li Y, Fan J, Wang Z, Zeng X, Sun Y, et al. The role of autophagy in the neurotoxicity of cationic PAMAM dendrimers. Biomaterials. 2014; 35: 7588-97.

25. Filomeni G, Desideri E, Cardaci S, Rotilio G, Ciriolo MR. Under the ROS...thiol network is the principal suspect for autophagy commitment. Autophagy. 2010; 6: 999-1005.

26. Filomeni G, De Zio D, Cecconi F. Oxidative stress and autophagy: the clash between damage and metabolic needs. Cell Death Differ. 2015; 22: 377-88.

27. Zhang J, Ney PA. Role of BNIP3 and NIX in cell death, autophagy, and mitophagy. Cell Death Differ. 2009; 16: 939-46.

28. Wiseman $H$, Halliwell $B$. Damage to DNA by reactive oxygen and nitrogen species: role in inflammatory disease and progression to cancer. Biochem J. 1996; 313 ( Pt 1): 17-29.

29. Li Y, Zeng X, Wang S, Sun Y, Wang Z, Fan J, et al. Inhibition of autophagy protects against PAMAM dendrimers-induced hepatotoxicity. Nanotoxicology. 2015; 9: 344-55.

30. Li Y, Zhu H, Zeng X, Fan J, Qian X, Wang S, et al. Suppression of autophagy enhanced growth inhibition and apoptosis of interferon-beta in human glioma cells. Mol Neurobiol. 2013; 47: 1000-10.

31. Petit AN, Debenest T, Eullaffroy P, Gagne F. Effects of a cationic PAMAM dendrimer on photosynthesis and ROS production of Chlamydomonas reinhardtii. Nanotoxicology. 2012; 6: 315-26.

32. Naha PC, Byrne HJ. Generation of intracellular reactive oxygen species and genotoxicity effect to exposure of nanosized polyamidoamine (PAMAM) dendrimers in PLHC-1 cells in vitro. Aquat toxicol. 2013; 132-133: 61-72.

33. Brinkkoetter PT, Song H, Losel R, Schnetzke U, Gottmann U, Feng Y, et al. Hypothermic injury: the mitochondrial calcium, ATP and ROS love-hate triangle out of balance. Cell Physiol Biochem. 2008; 22: 195-204.

34. Cui $\mathrm{H}$, Kong $\mathrm{Y}$, Zhang $\mathrm{H}$. Oxidative stress, mitochondrial dysfunction, and aging. J Signal Transduct. 2012; 2012: 646354.

35. Loos B, du Toit A, Hofmeyr JH. Defining and measuring autophagosome flux-concept and reality. Autophagy. 2014; 10: 2087-96.

36. Jiang P, Mizushima N. LC3- and p62-based biochemical methods for the analysis of autophagy progression in mammalian cells. Methods. 2015; 75: 13-8.

37. Xie Z, Xie Y, Xu Y, Zhou H, Xu W, Dong Q. Bafilomycin A1 inhibits autophagy and induces apoptosis in MG63 osteosarcoma cells. Mol Med Rep. 2014; 10: 1103-7.

38. Blommaart EF, Krause U, Schellens JP, Vreeling-Sindelarova H, Meijer AJ. The phosphatidylinositol 3-kinase inhibitors wortmannin and LY294002 inhibit autophagy in isolated rat hepatocytes. Eur J Biochem. 1997; 243: 240-6.

39. Pliyev BK, Menshikov M. Differential effects of the autophagy inhibitors 3-methyladenine and chloroquine on spontaneous and TNF-alpha-induced neutrophil apoptosis. Apoptosis. 2012; 17: 1050-65.

40. Zhang X, Chen S, Song L, Tang Y, Shen Y, Jia L, et al. MTOR-independent, autophagic enhancer trehalose prolongs motor neuron survival and ameliorates the autophagic flux defect in a mouse model of amyotrophic lateral sclerosis. Autophagy. 2014; 10: 588-602.

41. Spang N, Feldmann A, Huesmann H, Bekbulat F, Schmitt V, Hiebel C, et al. RAB3GAP1 and RAB3GAP2 modulate basal and rapamycin-induced autophagy. Autophagy. 2014; 10: 2297-309.

42. Saiki S, Sasazawa Y, Imamichi Y, Kawajiri S, Fujimaki T, Tanida I, et al. Caffeine induces apoptosis by enhancement of autophagy via $\mathrm{PI} 3 \mathrm{~K} / \mathrm{Akt} / \mathrm{mTOR} / \mathrm{p} 70 \mathrm{S6K}$ inhibition. Autophagy. 2011; 7: 176-87.

43. Jung $\mathrm{CH}$, Ro $\mathrm{SH}$, Cao J, Otto NM, Kim DH. mTOR regulation of autophagy. FEBS Lett. 2010; 584: 1287-95.

44. Shinojima N, Yokoyama $\mathrm{T}$, Kondo $\mathrm{Y}$, Kondo $\mathrm{S}$. Roles of the $\mathrm{Akt} / \mathrm{mTOR} / \mathrm{p} 70 \mathrm{~S} 6 \mathrm{~K}$ and ERK1/2 signaling pathways in curcumin-induced autophagy. Autophagy. 2007; 3: 635-7.

45. Zhong W, Zhu H, Sheng F, Tian Y, Zhou J, Chen Y, et al. Activation of the MAPK11/12/13/14 (p38 MAPK) pathway regulates the transcription of autophagy genes in response to oxidative stress induced by a novel copper complex in HeLa cells. Autophagy. 2014; 10: 1285-300.

46. Sadekar S, Ghandehari H. Transepithelial transport and toxicity of PAMAM dendrimers: implications for oral drug delivery. Adv Drug Deliv Rev. 2012; 64: 571-88.

47. Thiagarajan G, Greish K, Ghandehari H. Charge affects the oral toxicity of poly(amidoamine) dendrimers. Eur J Pharm Biopharm. 2013; 84: 330-4.

48. Thomas TP, Majoros I, Kotlyar A, Mullen D, Holl MM, Baker JR, Jr. Cationic poly(amidoamine) dendrimer induces lysosomal apoptotic pathway at therapeutically relevant concentrations. Biomacromolecules. 2009; 10: 3207-14.

49. Li Y, Wang S, Wang Z, Qian X, Fan J, Zeng X, et al. Cationic poly(amidoamine) dendrimers induced cyto-protective autophagy in hepatocellular carcinoma cells. Nanotechnology. 2014; 25: 365101.

50. Wan J, Wang JH, Liu T, Xie Z, Yu XF, Li W. Surface chemistry but not aspect ratio mediates the biological toxicity of gold nanorods in vitro and in vivo. Sci Rep. 2015; 5: 11398 .

51. Gorojod RM, Alaimo A, Porte Alcon S, Pomilio C, Saravia F, Kotler ML. The autophagic- lysosomal pathway determines the fate of glial cells under manganese- induced oxidative stress conditions. Free Radic Biol Med. 2015: FRBMD1500103.

52. Park WH. The effect of MAPK inhibitors and ROS modulators on cell growth and death of $\mathrm{H}(2) \mathrm{O}(2)$-treated HeLa cells. Mol Med Rep. 2013; 8: 557-64.
53. Roy B, Pattanaik AK, Das J, Bhutia SK, Behera B, Singh P, et al. Role of $\mathrm{PI} 3 \mathrm{~K} / \mathrm{Akt} / \mathrm{mTOR}$ and MEK/ERK pathway in Concanavalin A induced autophagy in HeLa cells. Chem Biol Interact. 2014; 210: 96-102.

54. Cagnol S, Chambard JC. ERK and cell death: mechanisms of ERK-induced cell death--apoptosis, autophagy and senescence. FEBS J. 2010; 277: 2-21. 